
تأثير الأثيفون في بعض دلالات النمو الفسيولوجية والتركيب المعدني لنبات الحلبة

\author{
سعد الدين ماجد الحفوظي عزوان قاسم حسن الطائي

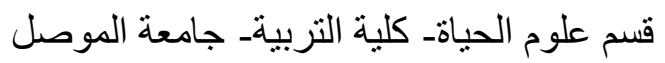 \\ الخلاصة
}

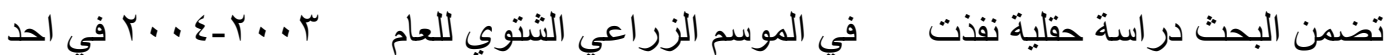

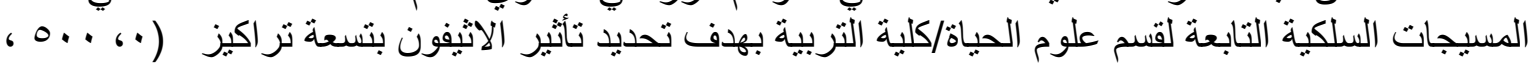
ال

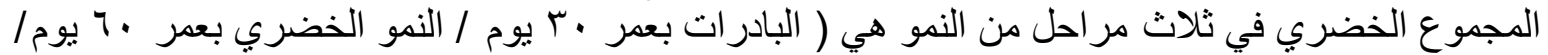

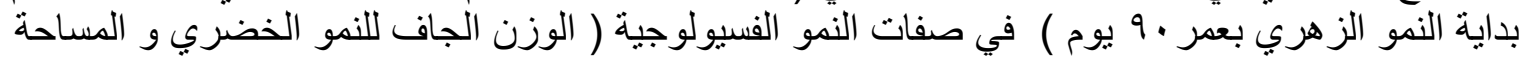

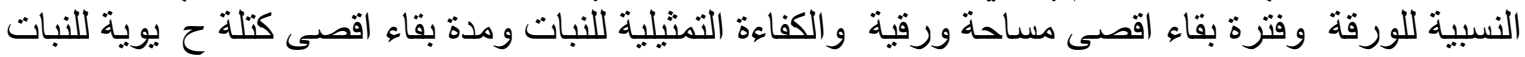

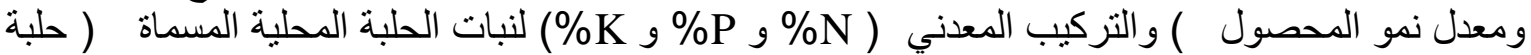

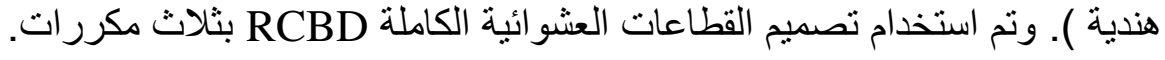

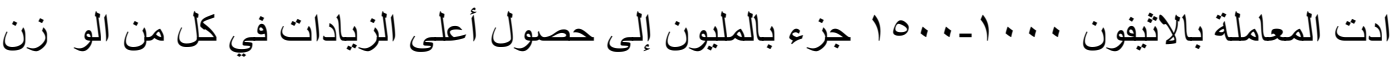

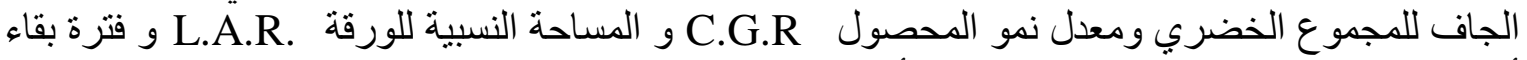

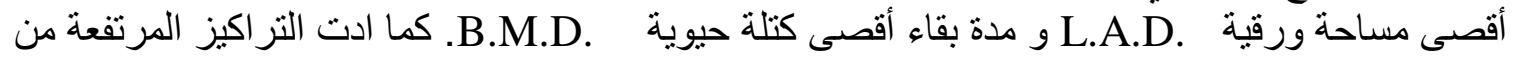

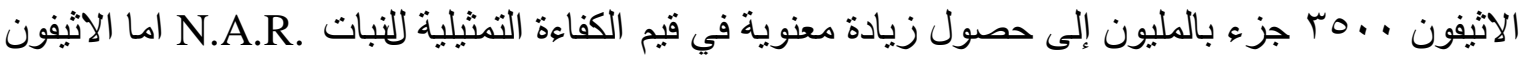

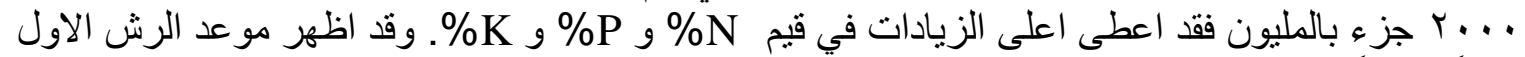
تفوقاً معنوياً على بقية المو اعيد.

\title{
المقدمة
}

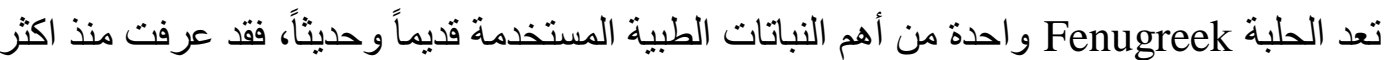

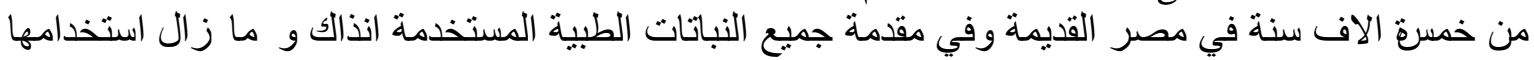

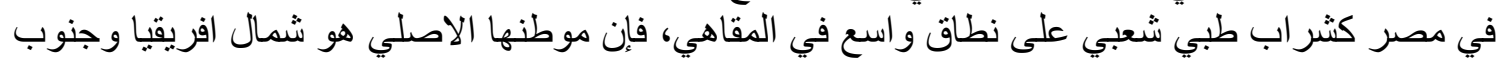

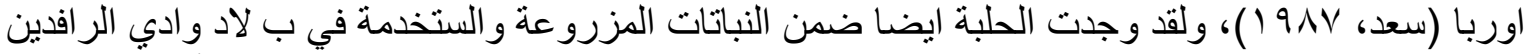

حسب ما جاء في قائمة النباتات الاروماتية المدونة في المكتبة الكبرى في نينوى في عهد الملك آثنور بانيبات النيال

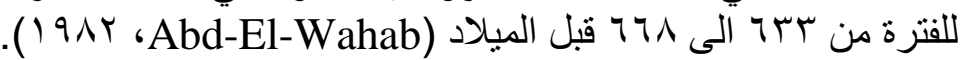

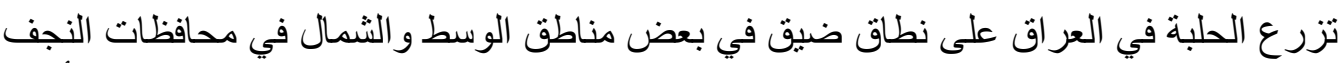

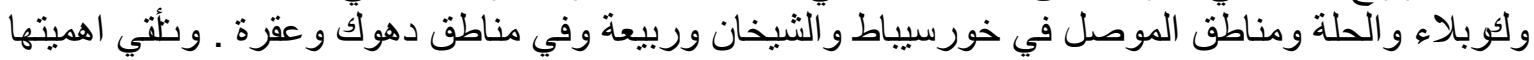

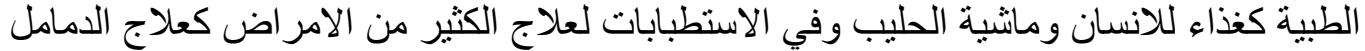

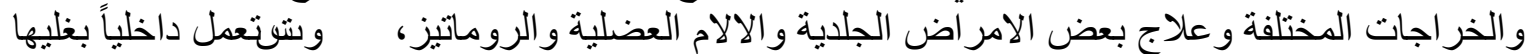

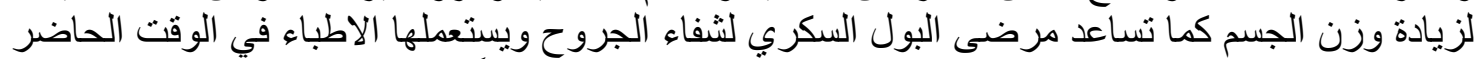

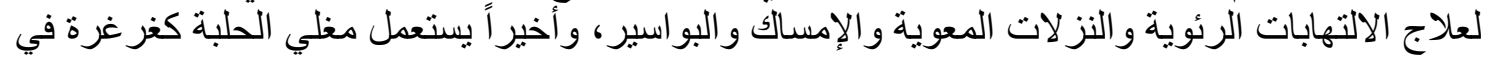

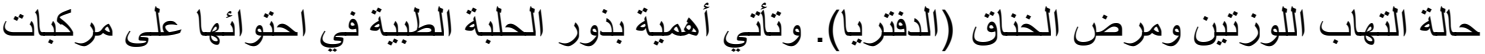

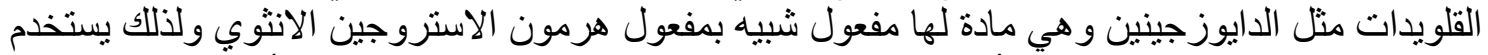

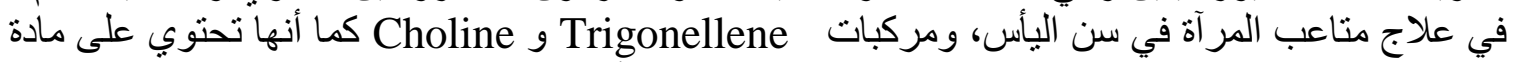
Trimethylanin

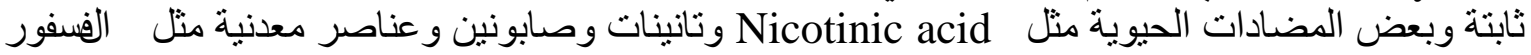

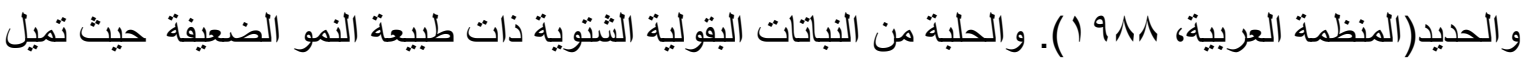

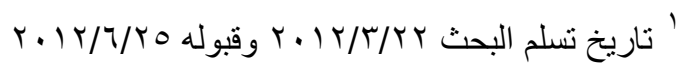


للاضطجاع وخاصة في حالة زيادة الرطوبة وضعف او قلة فترات السطوع الثمسي و ارتفاع الحرارة خلال

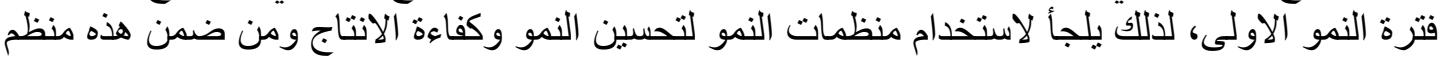

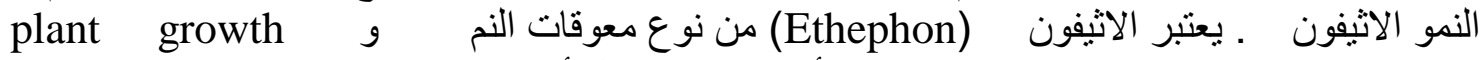

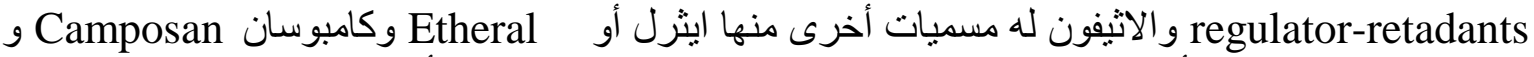

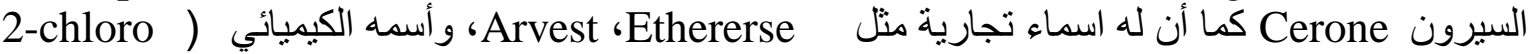
(ethyl phosphonic acid

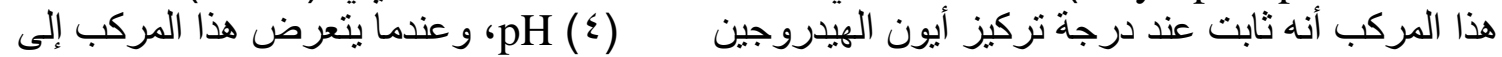

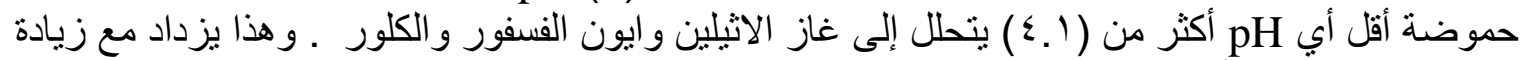

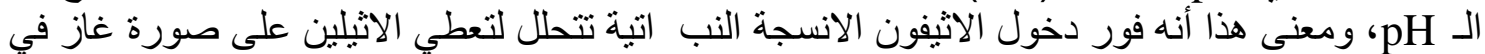

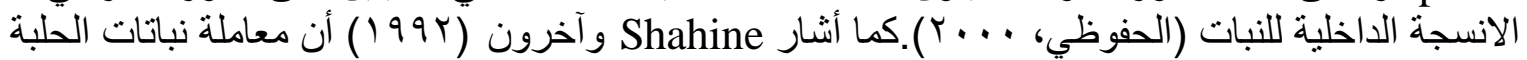

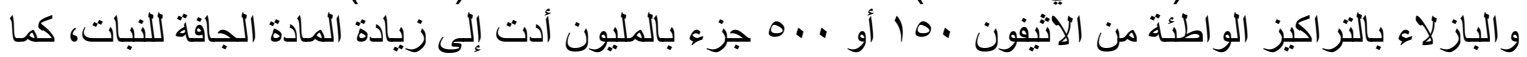

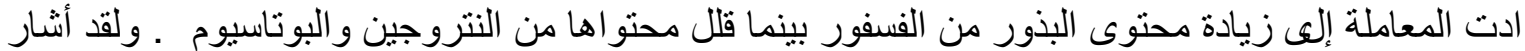

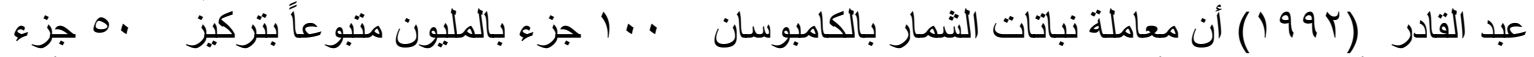

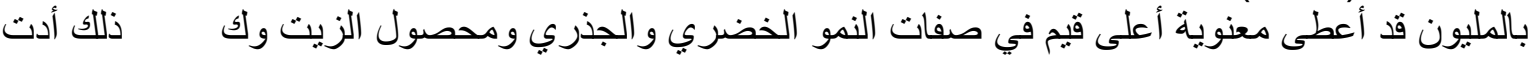
المعاملة إلى زيادة معنوية في محصول البذرة لكل نبات و النسبة المئوية للكاربو هيدر ات الكية الكلية و النايتروجين

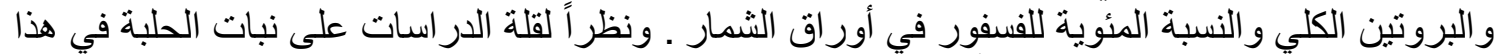

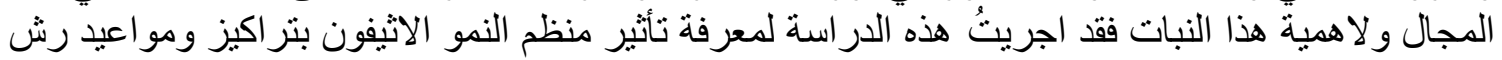
مختلفة على النمو والتركيب المعدني لنبات الحلبة الصنف المحلي الصني المسمى حلبة هندية. مواد وطرائق البحث

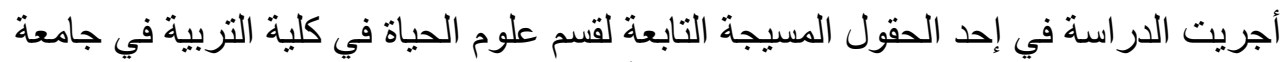

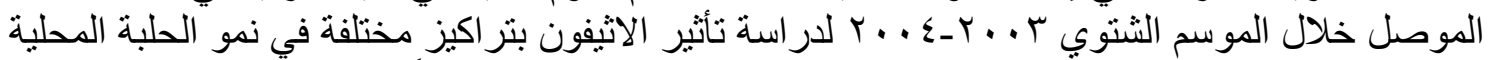
المسماة (حلبة هندية) Trigonella foenum-graecum L وحاصلها رشاً على المجموع الخضري لثناث

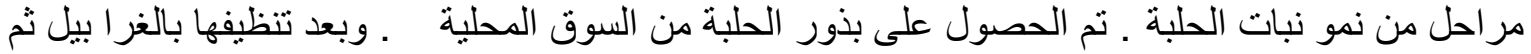

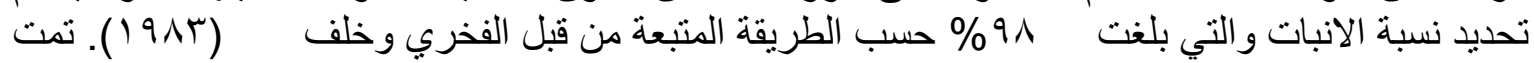

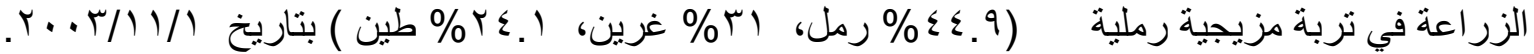

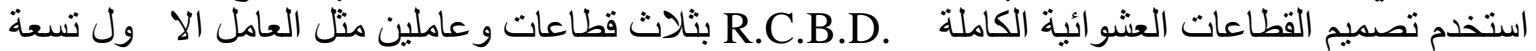

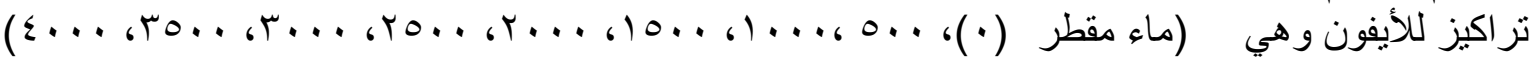

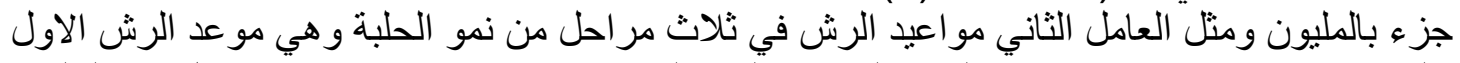

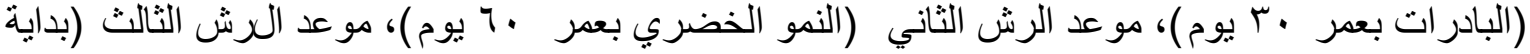

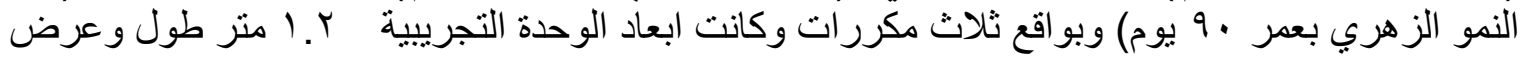

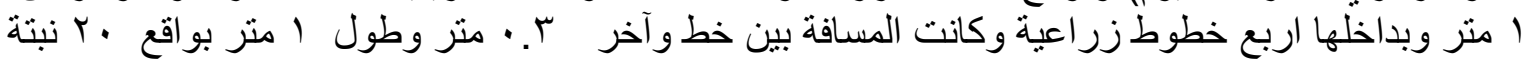

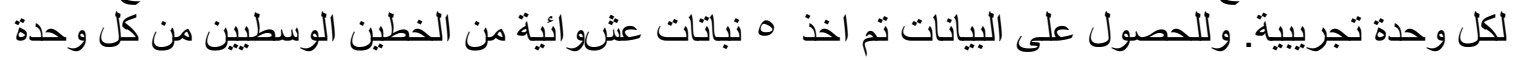

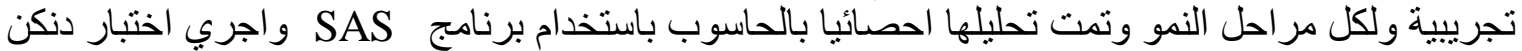

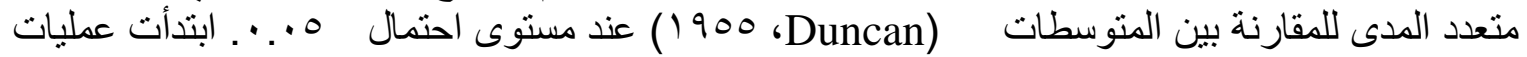

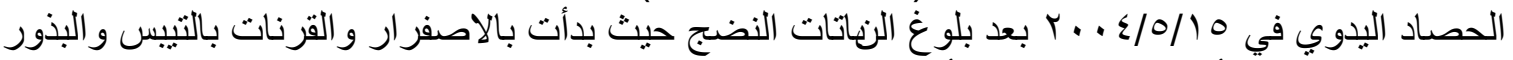

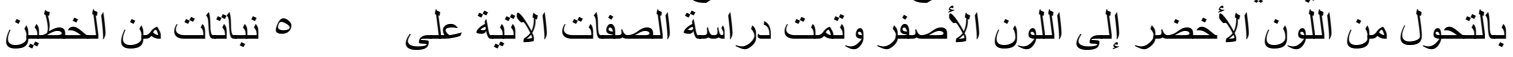
الوسطيين لكل وحدة تجريبية.

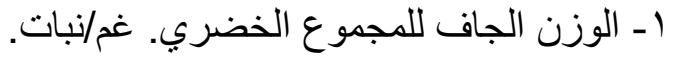
بالإضافة إلى أعلاه تم قياس الصفات التالية و بلاعتماد على بلى بيانات القر اعتين الأولى و الثالثة r- المساحة الورقية L.A. سمَّ) حسبت المساحة الورقية بتطبيق المعادلة التالية:

مساحة · ـ أقر اص (ملمَّ) X وزن الور اق الجافة (ملغم)

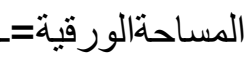

$$
\text { وزن · · أقر اص (ملغم) }
$$




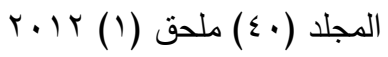

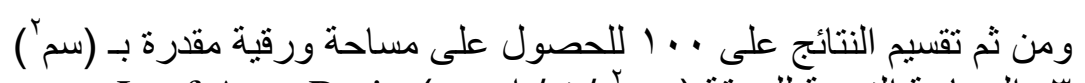

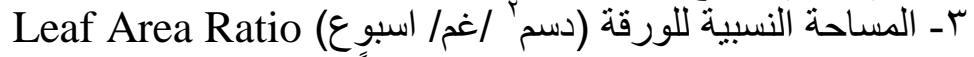
وحسبت المساحة النسبية للورقة اعتماداً على طريقة Radford (19 (1 ) وكما في المعادلة التالية:

$$
\text { L.A.R }=\frac{\log \mathrm{W}_{2}-\log \mathrm{W}_{1}}{\log \mathrm{A}_{2}-\log \mathrm{A}_{1}} \times \frac{\left(\mathrm{LA}_{2}-\mathrm{LA}_{1}\right)}{\left(\mathrm{W}_{2}-\mathrm{W}_{1}\right)}
$$

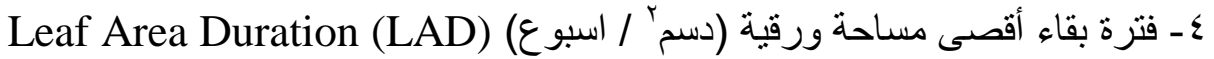

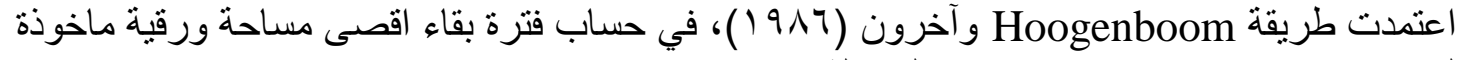

L.A.D. $=\frac{\left(\mathrm{t}_{2}-\mathrm{t}_{1}\right) \times\left(\mathrm{LA}_{2}+\mathrm{LA}_{1}\right)}{2}$ من المصدر Harper (9VV) (1 ) وكما في المعادلة

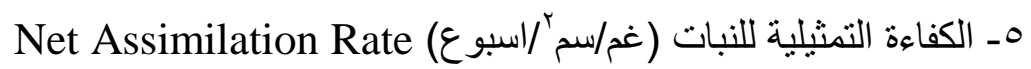
وتم حساب الكفاءة التمثيلية للنبات حسب طريقة Radford (197V) (1 ) وكما في المعادلة التالية: $\mathrm{NAR}=\frac{\mathrm{W}_{2}-\mathrm{W}_{1}}{\mathrm{LA}_{2}-\mathrm{LA}_{1}} \times \frac{\log \mathrm{LA}_{2}-\log \mathrm{LA}_{1}}{\left(\mathrm{t}_{2-} \mathrm{t}_{1}\right)}$

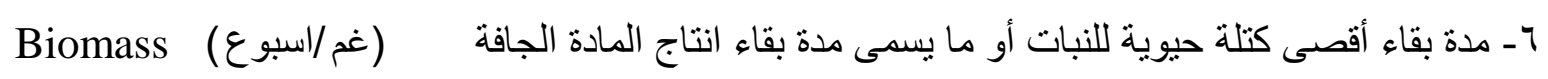
.Duration وحسبت بطريقة (1978) Hunt مأخوذة من المصدر عيسى (.999) كما في المعادلة التالية: B.M.D. $=\frac{\mathrm{W}_{2}+\mathrm{W}_{1}}{2} \times\left(\mathrm{t}_{2-} \mathrm{t}_{1}\right)$

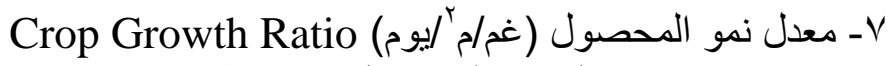

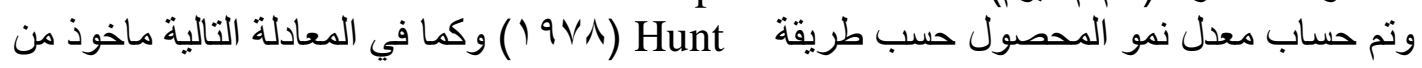

C.G.R $=\frac{1}{G A} \times-\frac{\left(\mathrm{W}_{2}-\mathrm{W}_{1}\right)}{\mathrm{t}_{2-} \mathrm{t}_{1}}$ المصدر عيسى (999 (199) :

$$
\begin{aligned}
& \mathrm{t}_{2-} \mathrm{t}_{1} \\
& \text { = LA }
\end{aligned}
$$

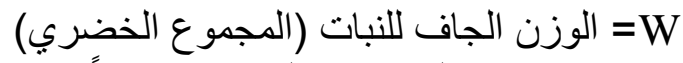

$$
\begin{aligned}
& \text { (t }=\left(t_{2}-t_{1}\right) \\
& \text { GA } \\
& \text { 1. لو غارتئ = Log } \\
& \text { التركيب المعدني للنبات }
\end{aligned}
$$

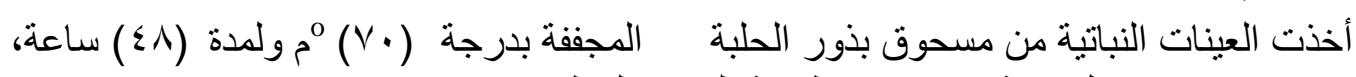

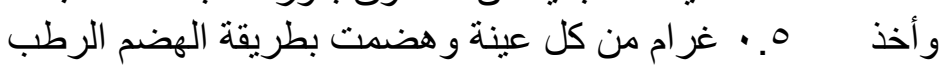

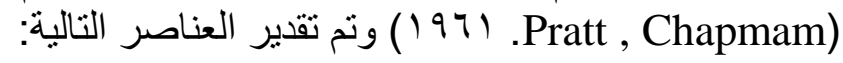

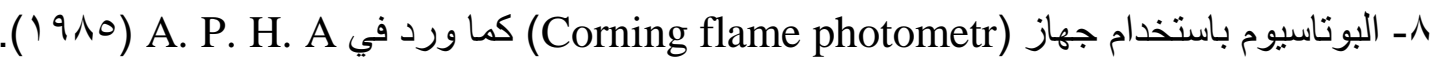

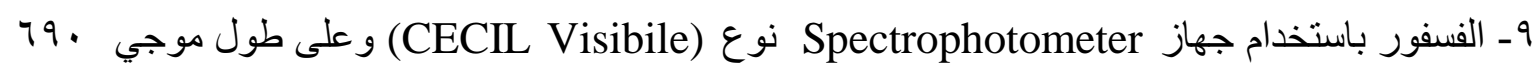
نانوميتر. كما ورد في A. A. P. A. A (1910).

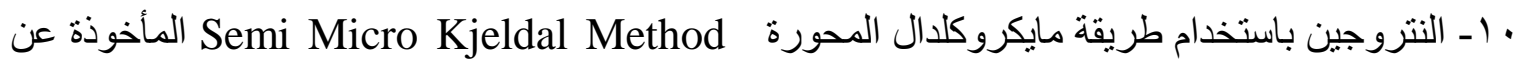


الجدول ( ) درجات الحرارة الصغرى و العظمى والمعدل الثهري وكم ية الامطار ومعدل الرطوبة النسبية

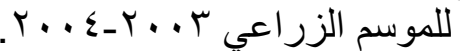

\begin{tabular}{|c|c|c|c|c|c|}
\hline الثألثهري & للارطوبة النسبية & لالدعدل الثهري الحرارة & لدرجة الحرل الثهري & الثهر & السنة \\
\hline Nז.o & זד & rI. & $\vee . \wedge$ & الثنرين & $r \ldots r$ \\
\hline$\Delta r . \cdot$ & NT & $1 \varepsilon .1$ & $0 . r$ & كانون الأول & $r \ldots r$ \\
\hline$\Lambda V_{.}$. & $\Lambda$. & 11.0 & 0.1 & كانون الثاني & $r \ldots \varepsilon$ \\
\hline $7 .$. & Vo & $1 \leqslant . Y$ & $\varepsilon .1$ & شُباط & $r \ldots \varepsilon$ \\
\hline T.7 & $7 r$ & Yr.O & $V . V$ & آذار & $r \ldots \varepsilon$ \\
\hline$V 0.1$ & OV & ro.1 & 1.0 & نيسان & $r \ldots \varepsilon$ \\
\hline$\varepsilon .7$ & $\varepsilon \wedge$ & TY.O & 17.1 & أيار & $r \ldots \varepsilon$ \\
\hline- & r & rq. V & YI. & حزيران & $r \ldots \varepsilon$ \\
\hline
\end{tabular}

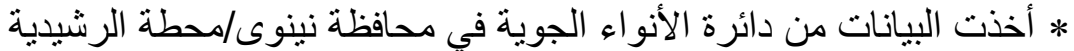

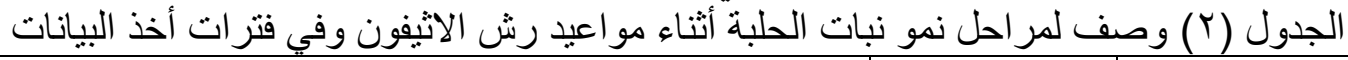

\begin{tabular}{|c|c|c|}
\hline وصف مرحلة النمو & ت تاريخها & المعاملة \\
\hline & $r \ldots r / l / l$ & الزراعة \\
\hline النبات في طور البادرة، وبعمر شهر من الزر اعة يحتوي النبات & $r \ldots r / r / l$ & 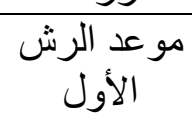 \\
\hline 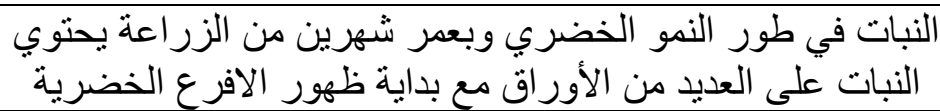 & $r \ldots \varepsilon / / / 1$ & موعد الرش الثاني \\
\hline 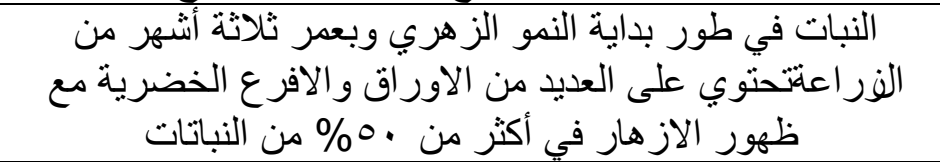 & $r \ldots \varepsilon / r / T$ & موعد الرش الثنالث \\
\hline 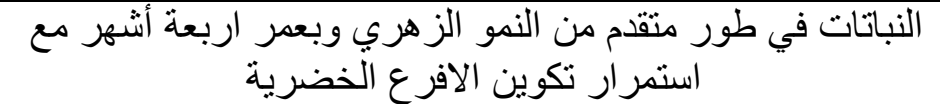 & $r \ldots \varepsilon / \Gamma / 1$ & مو الاولى القر اعة \\
\hline 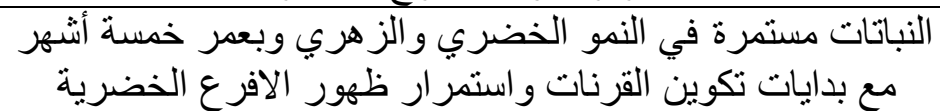 & $r \ldots \varepsilon / \varepsilon / 1$ & مو عد القراعة الثانية \\
\hline 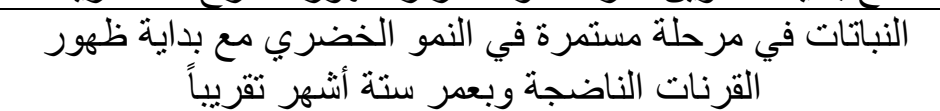 & $r \ldots \varepsilon / 0 / 1$ & مو عد القر اعة \\
\hline
\end{tabular}

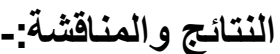

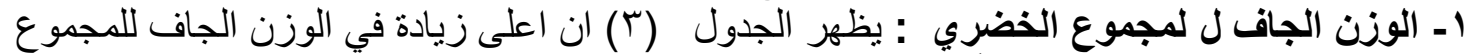

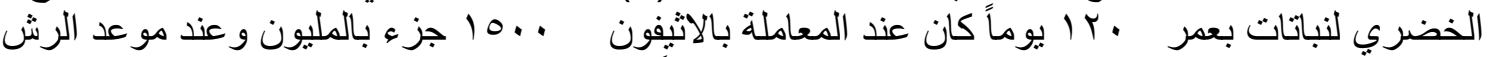

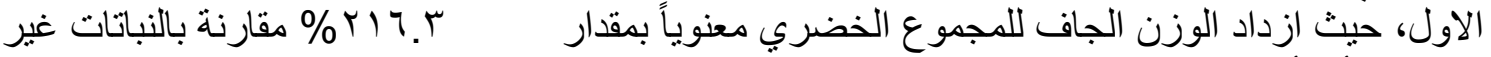

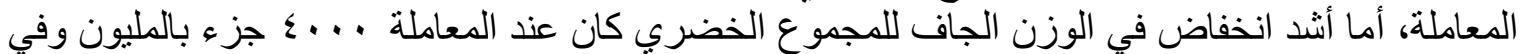

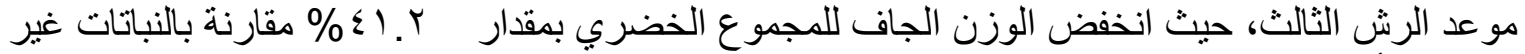

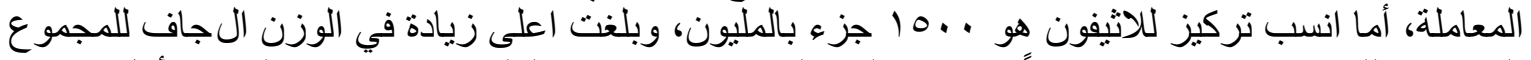

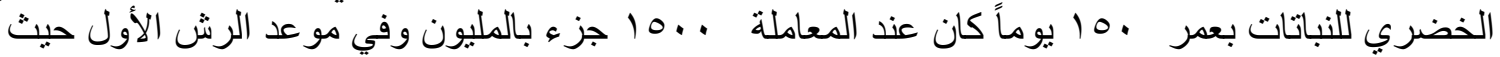

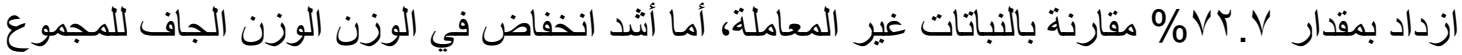

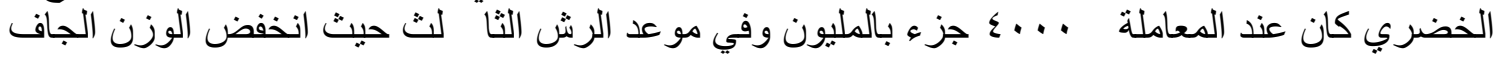




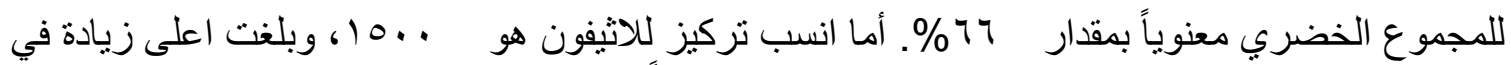

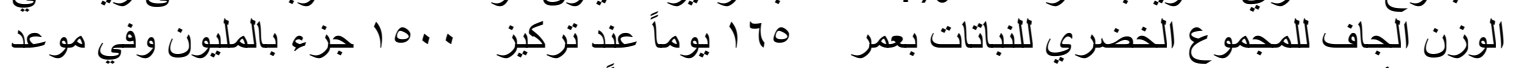

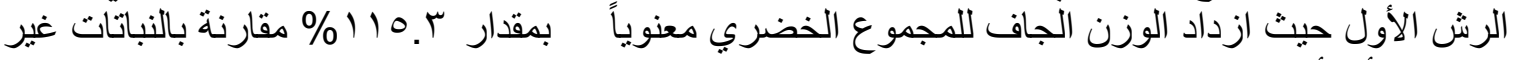

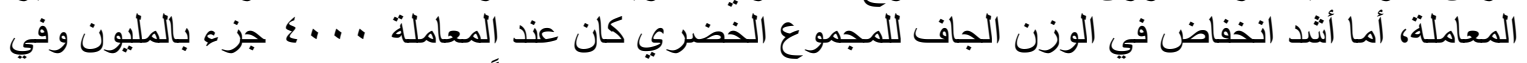

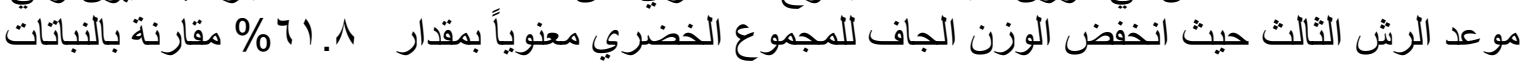

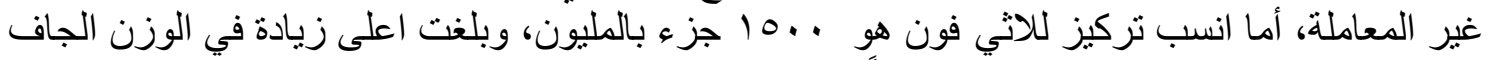

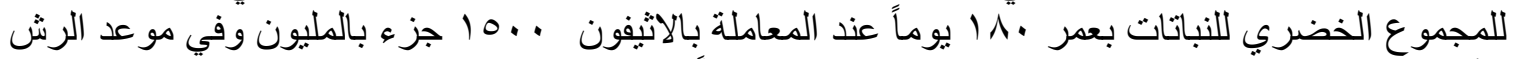

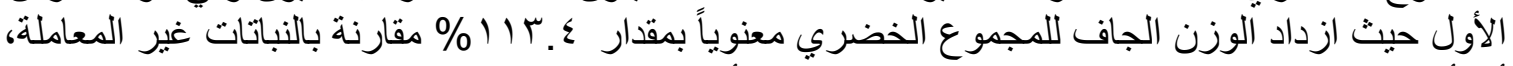

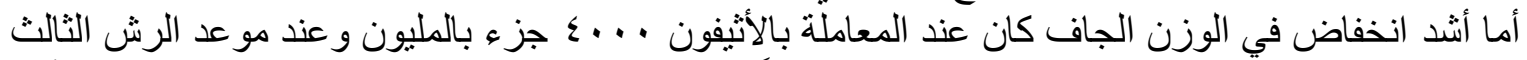

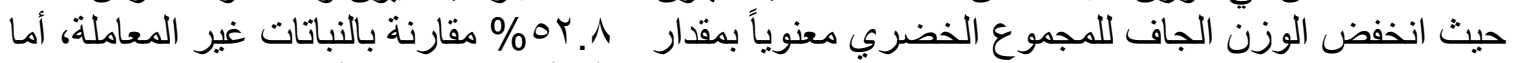

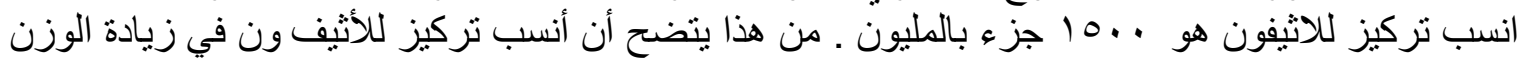

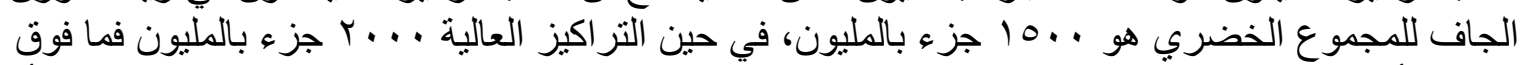

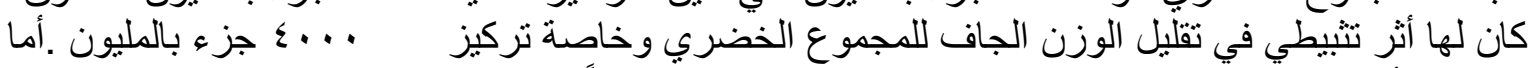

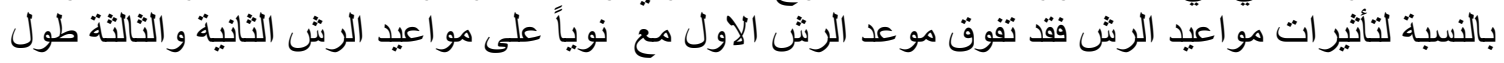
فترة نمو النبات.

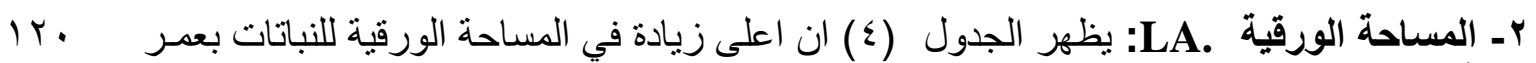

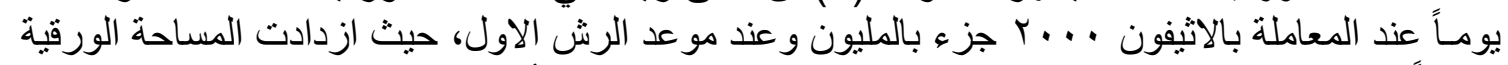

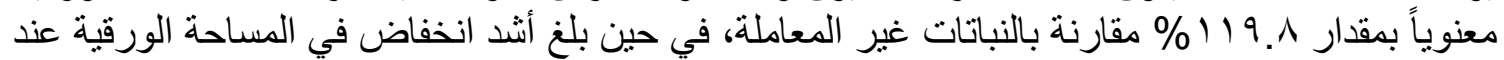

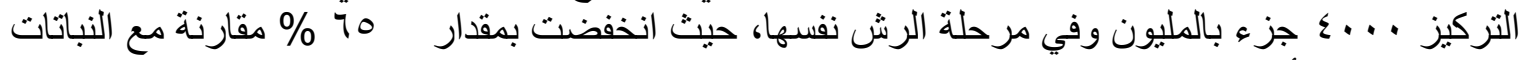

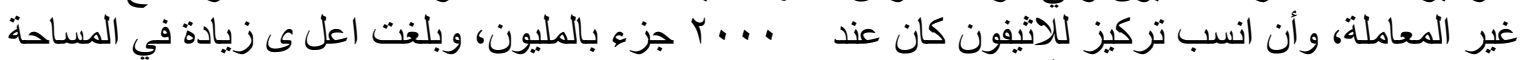

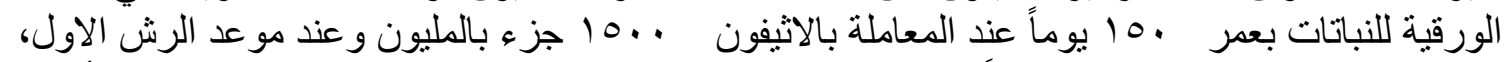

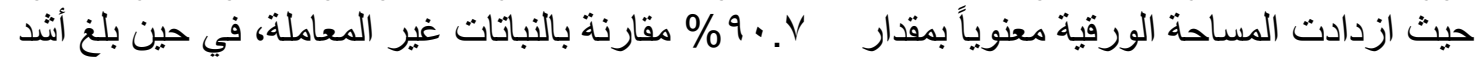

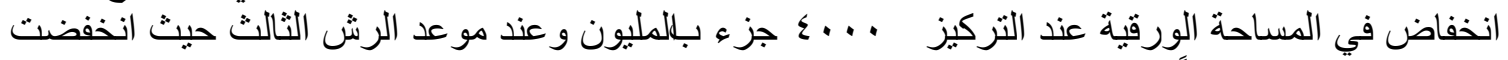

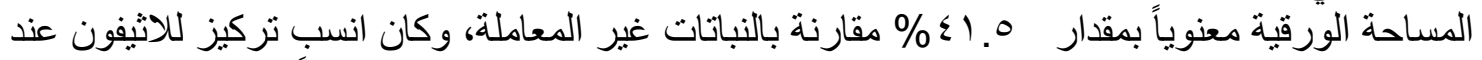

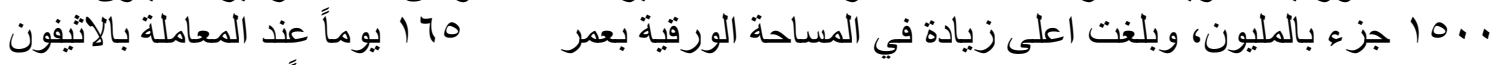

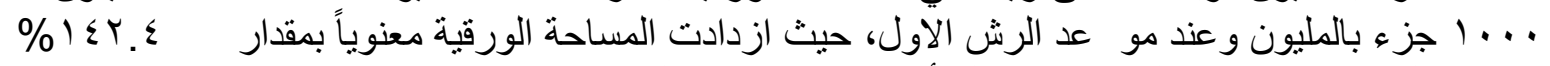

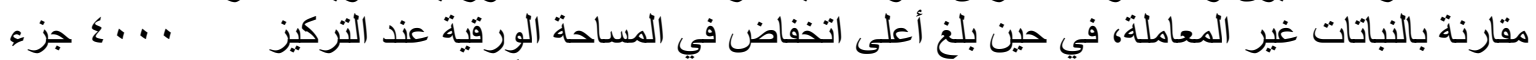

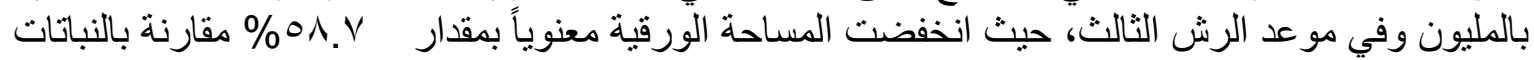

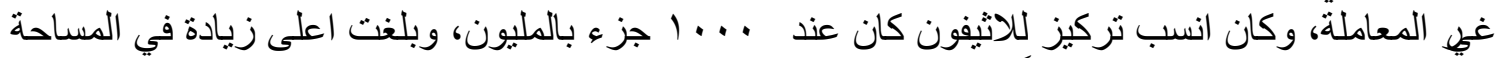

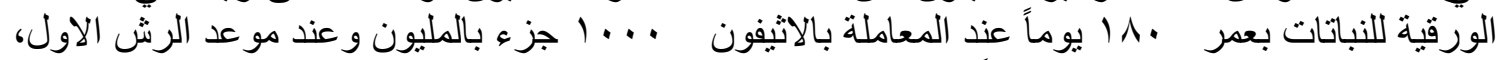

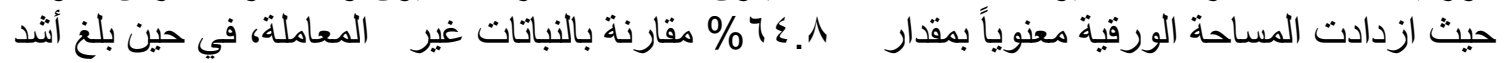

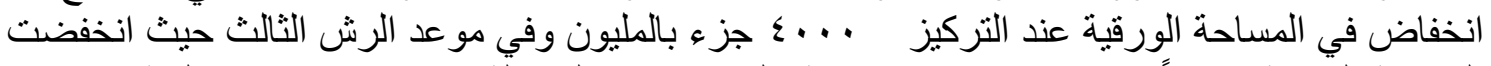

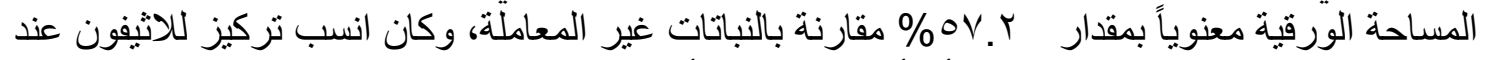

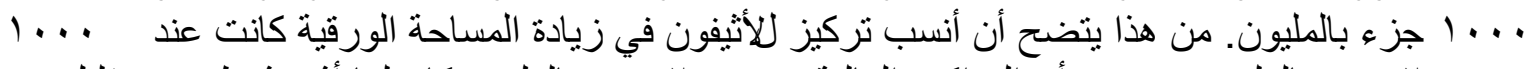

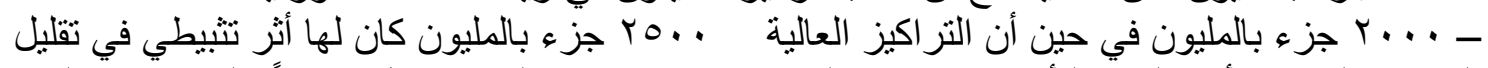

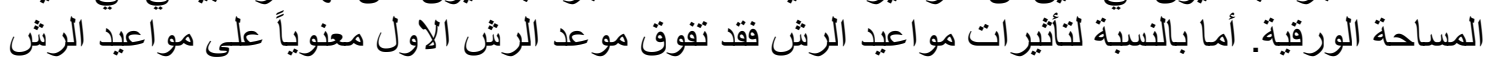
الثانية و الثالثة.

إن تأثير الاثثفون في خفض المساحة الورقية ناتج من تأثثيره في خفض حجم الخلايا وتقليل المسافات

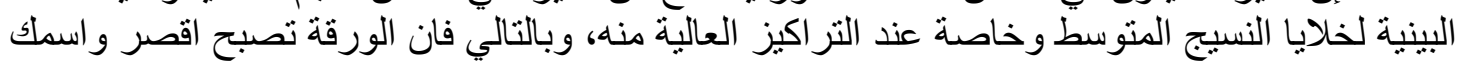

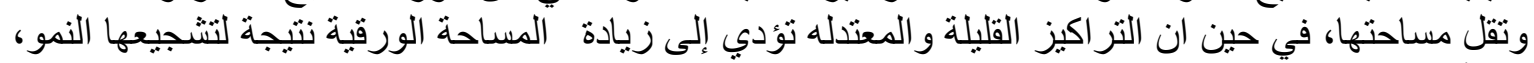

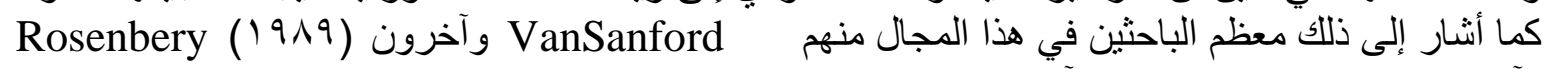

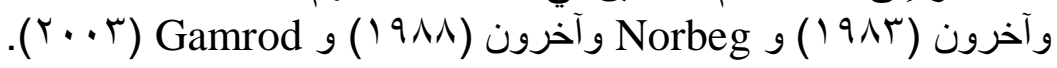




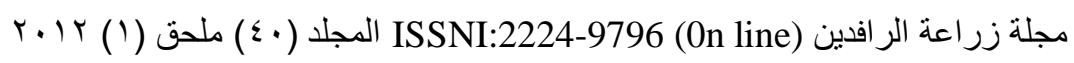
ISSN:1815-316X (Print)

جدول (ب) تأثير نر اكيز ومو اعيد رش الاثثفون في الوزن الجاف للمجموع الخضري غم / نبات

\begin{tabular}{|c|c|c|c|c|c|c|c|c|c|c|c|c|}
\hline \multicolumn{4}{|c|}{ القر اعة الثالثة بعمر ، ^ا يوماً } & \multicolumn{4}{|c|}{ القر اءة الثانية بعمر ـ10 يوماً } & \multicolumn{4}{|c|}{ القر اعة الأولى بعمر · با يوماً } & \multirow{2}{*}{ الاثيفونيز } \\
\hline للتر اكيز & الثالثة & الثانية & الأولى & للتر اكي & الثالثة & الثانية & الأولى & للتزر اكي & الثالثة & الثانية & الأرُشَ & \\
\hline $\begin{array}{c}17.10 \\
\mathrm{de}\end{array}$ & $\begin{array}{c}17.88 \\
\mathrm{~g}-\mathrm{j}\end{array}$ & $\begin{array}{c}17.46 \\
\mathrm{e}-\mathrm{j}\end{array}$ & $\begin{array}{c}16.57 \\
\mathrm{~g}-\mathrm{k}\end{array}$ & $\begin{array}{c}6.520 \\
\mathrm{de}\end{array}$ & $\begin{array}{c}6.300 \\
e-i\end{array}$ & $\begin{array}{c}6.670 \\
\mathrm{~d}-\mathrm{g}\end{array}$ & $\begin{array}{c}6.590 \\
\mathrm{~d}-\mathrm{g}\end{array}$ & $\begin{array}{c}2.184 \\
\mathrm{~d}\end{array}$ & $\begin{array}{c}2.063 \\
\text { hij }\end{array}$ & $\begin{array}{c}2.393 \\
\mathrm{~g}-\mathrm{j}\end{array}$ & $\begin{array}{c}2.096 \\
\text { hij }\end{array}$ & . \\
\hline $\begin{array}{c}21.17 \\
\mathrm{c}\end{array}$ & $\begin{array}{c}19.03 \\
\mathrm{~d}-\mathrm{i}\end{array}$ & $\begin{array}{c}21.21 \\
\mathrm{~d}-\mathrm{h}\end{array}$ & $\begin{array}{c}23.28 \\
\text { b-f }\end{array}$ & $\begin{array}{c}7.292 \\
\text { bcd }\end{array}$ & $\begin{array}{c}6.610 \\
\mathrm{~d}-\mathrm{g}\end{array}$ & $\begin{array}{c}7.303 \\
\mathrm{c}-\mathrm{g}\end{array}$ & $\begin{array}{c}7.963 \\
b-f\end{array}$ & $\begin{array}{c}3.766 \\
\text { bc }\end{array}$ & $\begin{array}{c}2.643 \\
f-j\end{array}$ & $\begin{array}{c}4.116 \\
\mathrm{c}-\mathrm{h}\end{array}$ & $\begin{array}{c}4.540 \\
b-f\end{array}$ & $0 .$. \\
\hline $\begin{array}{c}24.36 \\
b\end{array}$ & $\begin{array}{c}21.32 \\
\mathrm{~d}-\mathrm{h}\end{array}$ & $\begin{array}{c}24.06 \\
\text { bcd }\end{array}$ & $\begin{array}{c}27.72 \\
\text { bc }\end{array}$ & $\begin{array}{c}8.402 \\
\text { bc }\end{array}$ & $\begin{array}{c}7.723 \\
b-g\end{array}$ & $\begin{array}{c}8.293 \\
\text { b-e }\end{array}$ & $\begin{array}{c}9.190 \\
a-d\end{array}$ & $\begin{array}{c}4.690 \\
\mathrm{ab}\end{array}$ & $\begin{array}{c}3.300 \\
\mathrm{~d}-\mathrm{j}\end{array}$ & $\begin{array}{c}4.500 \\
b-g\end{array}$ & $\begin{array}{c}6.270 \\
\mathrm{ab}\end{array}$ & $1 \ldots$ \\
\hline $\begin{array}{c}29.58 \\
\mathrm{a}\end{array}$ & $\begin{array}{c}23.96 \\
\text { bcd }\end{array}$ & $\begin{array}{c}29.25 \\
b\end{array}$ & $\begin{array}{c}35.36 \\
\mathrm{a}\end{array}$ & $\begin{array}{c}9.890 \\
\mathrm{a}\end{array}$ & $\begin{array}{c}8.580 \\
\text { b-e }\end{array}$ & $\begin{array}{c}9.723 \\
\text { abc }\end{array}$ & $\begin{array}{c}11.38 \\
\mathrm{a}\end{array}$ & $\begin{array}{c}5.255 \\
\mathrm{a}\end{array}$ & $\begin{array}{c}3.920 \\
c-j\end{array}$ & $\begin{array}{c}5.216 \\
a-d\end{array}$ & $\begin{array}{c}6.630 \\
\mathrm{a}\end{array}$ & $10 \ldots$ \\
\hline $\begin{array}{c}25.52 \\
b\end{array}$ & $\begin{array}{c}23.61 \\
\text { b-e }\end{array}$ & $\begin{array}{c}23.20 \\
b-f\end{array}$ & $\begin{array}{c}28.94 \\
\text { b }\end{array}$ & $\begin{array}{c}8.538 \\
b\end{array}$ & $\begin{array}{c}6.526 \\
\text { d-h }\end{array}$ & $\begin{array}{c}8.890 \\
\text { a-d }\end{array}$ & $\begin{array}{c}10.11 \\
\mathrm{ab}\end{array}$ & $\begin{array}{c}4.646 \\
\mathrm{ab}\end{array}$ & $\begin{array}{c}3.333 \\
\mathrm{~d}-\mathrm{j}\end{array}$ & $\begin{array}{c}4.810 \\
\text { a-e }\end{array}$ & $\begin{array}{c}5.796 \\
\text { abc }\end{array}$ & $r \ldots$ \\
\hline $\begin{array}{c}20.23 \\
\mathrm{~cd}\end{array}$ & $\begin{array}{c}15.86 \\
\text { g-k }\end{array}$ & $\begin{array}{c}19.63 \\
\mathrm{~d}-\mathrm{i}\end{array}$ & $\begin{array}{c}25.20 \\
\text { bcd }\end{array}$ & $\begin{array}{c}7.101 \\
\text { cde }\end{array}$ & $\begin{array}{c}5.090 \\
\text { g-j }\end{array}$ & $\begin{array}{c}7.683 \\
b-g\end{array}$ & $\begin{array}{c}8.530 \\
\text { b-e }\end{array}$ & $\begin{array}{c}3.818 \\
\text { bc }\end{array}$ & $\begin{array}{c}2.473 \\
\mathrm{f}-\mathrm{j}\end{array}$ & $\begin{array}{c}4.046 \\
\mathrm{c}-\mathrm{h}\end{array}$ & $\begin{array}{c}0.936 \\
\text { a-d }\end{array}$ & ro.. \\
\hline $\begin{array}{c}17.07 \\
\mathrm{de}\end{array}$ & $\begin{array}{c}12.67 \\
\mathrm{jkl}\end{array}$ & $\begin{array}{c}16.49 \\
\text { g-k }\end{array}$ & $\begin{array}{c}25.05 \\
\mathrm{c}-\mathrm{g}\end{array}$ & $\begin{array}{c}5.803 \\
\text { ef }\end{array}$ & $\begin{array}{c}3.836 \\
\mathrm{ijk}\end{array}$ & $\begin{array}{c}6.130 \\
e-i\end{array}$ & $\begin{array}{c}7.443 \\
\mathrm{c}-\mathrm{g}\end{array}$ & $\begin{array}{c}3.156 \\
\mathrm{~cd}\end{array}$ & $\begin{array}{c}1.900 \\
\mathrm{ij}\end{array}$ & $\begin{array}{c}3.183 \\
\mathrm{~d}-\mathrm{j}\end{array}$ & $\begin{array}{c}4.386 \\
b-g\end{array}$ & r... \\
\hline $\begin{array}{c}15.47 \\
\mathrm{e}\end{array}$ & $\begin{array}{c}10.93 \\
\mathrm{kl}\end{array}$ & $\begin{array}{c}14.57 \\
\text { ijk }\end{array}$ & $\begin{array}{c}20.92 \\
\text { d-h }\end{array}$ & $\begin{array}{c}5.044 \\
\mathrm{fg}\end{array}$ & $\begin{array}{c}3.283 \\
\mathrm{jk}\end{array}$ & $\begin{array}{c}5.156 \\
g-j\end{array}$ & $\begin{array}{c}6.693 \\
\mathrm{a}-\mathrm{g}\end{array}$ & $\begin{array}{c}2.461 \\
\mathrm{~d}\end{array}$ & $\begin{array}{c}1.440 \\
\mathrm{j}\end{array}$ & $\begin{array}{c}2.693 \\
e-j\end{array}$ & $\begin{array}{c}3.250 \\
\mathrm{~d}-\mathrm{j}\end{array}$ & ro... \\
\hline $\begin{array}{c}12.00 \\
\mathrm{f}\end{array}$ & $\begin{array}{c}8.440 \\
1\end{array}$ & $\begin{array}{c}12.28 \\
\mathrm{jkl}\end{array}$ & $\begin{array}{c}15.27 \\
\mathrm{~h}-\mathrm{k}\end{array}$ & $\begin{array}{c}3.885 \\
\mathrm{~g}\end{array}$ & $\begin{array}{c}2.140 \\
\mathrm{k}\end{array}$ & $\begin{array}{c}4.000 \\
\text { h-k }\end{array}$ & $\begin{array}{c}5.516 \\
\mathrm{f}-\mathrm{j}\end{array}$ & $\begin{array}{c}2.137 \\
\mathrm{~d}\end{array}$ & $\begin{array}{c}1.213 \\
\mathrm{~J}\end{array}$ & $\begin{array}{c}2.413 \\
\mathrm{f}-\mathrm{j}\end{array}$ & $\begin{array}{c}2.786 \\
e-j\end{array}$ & $\varepsilon \ldots$ \\
\hline & $\begin{array}{c}17.01 \\
\mathrm{c}\end{array}$ & $\begin{array}{c}19.79 \\
b\end{array}$ & $\begin{array}{c}23.92 \\
\mathrm{a}\end{array}$ & & $\begin{array}{c}5.565 \\
\mathrm{c}\end{array}$ & $\begin{array}{c}7.104 \\
\mathrm{~b}\end{array}$ & $\begin{array}{c}8.157 \\
\mathrm{a}\end{array}$ & & $\begin{array}{c}2.476 \\
\mathrm{c}\end{array}$ & $\begin{array}{c}3.708 \\
\text { b }\end{array}$ & $\begin{array}{c}4.521 \\
\mathrm{a}\end{array}$ & للرش المعدل \\
\hline
\end{tabular}

المعدلات التي تثترك بنفس الأحرف لكل عمود لا تختلف معنويا حسب اختبار دنكن متعدد الحدود عند مستوى احتماله\% 


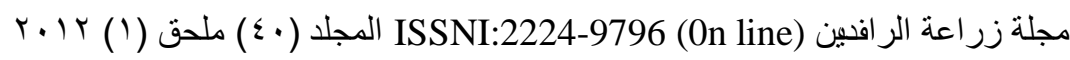
ISSN:1815-316X (Print)

الجدول (ع) نأثثير تر اكيز ومو اعيد رش الاثيفون في المساحة الورقية سمَ / نبات

\begin{tabular}{|c|c|c|c|c|c|c|c|c|c|c|c|c|}
\hline \multicolumn{4}{|c|}{ القر اءة الثالثة بعمر ، ا يوماً } & \multicolumn{4}{|c|}{ القر اءة الثانية بعمر ـ ا يوماً } & \multicolumn{4}{|c|}{ القر اعة الأولى بعمر · با يوماً } & \multirow{2}{*}{$\begin{array}{c}\text { تركيز } \\
\text { الاثيفون } \\
\text { ppm }\end{array}$} \\
\hline للتر اكيز & الثالثة & الثانية & الأرشلى & للتر اكيز & الثالثة & الثانية & الأوشى & للتر اكيز & الثالثة & الثانية & الأرشى & \\
\hline $\begin{array}{c}675.3 \\
c\end{array}$ & $\begin{array}{c}689.9 \\
\text { e-h }\end{array}$ & $\begin{array}{c}660.0 \\
f-j\end{array}$ & $\begin{array}{c}675.9 \\
\mathrm{e}-\mathrm{i}\end{array}$ & $\begin{array}{c}401.1 \\
\text { bed }\end{array}$ & $\begin{array}{c}377.2 \\
c-f\end{array}$ & $\begin{array}{c}425.9 \\
b-f\end{array}$ & $\begin{array}{c}400.1 \\
b-f\end{array}$ & $\begin{array}{c}230.9 \\
\mathrm{~cd}\end{array}$ & $\begin{array}{c}224.2 \\
\text { e-h }\end{array}$ & $\begin{array}{c}239.1 \\
\text { e-h }\end{array}$ & $\begin{array}{c}229.5 \\
\text { e-h }\end{array}$ & • \\
\hline $\begin{array}{c}889.7 \\
\text { b }\end{array}$ & $\begin{array}{c}772.7 \\
\mathrm{c}-\mathrm{g}\end{array}$ & $\begin{array}{c}911.5 \\
\text { a-d }\end{array}$ & $\begin{array}{c}984.8 \\
\text { abc }\end{array}$ & $\begin{array}{c}446.2 \\
\text { bc }\end{array}$ & $\begin{array}{c}413.2 \\
b-f\end{array}$ & $\begin{array}{c}472.7 \\
b-f\end{array}$ & $\begin{array}{c}452.7 \\
b-f\end{array}$ & $\begin{array}{c}270.7 \\
\text { bc }\end{array}$ & $\begin{array}{c}236.3 \\
\text { e-h }\end{array}$ & $\begin{array}{c}263.8 \\
\mathrm{~d}-\mathrm{g}\end{array}$ & $\begin{array}{c}312.2 \\
b-f\end{array}$ & $0 .$. \\
\hline $\begin{array}{c}1042 \\
\mathrm{a}\end{array}$ & $\begin{array}{c}931.5 \\
\text { a-d }\end{array}$ & $\begin{array}{c}1081 \\
\mathrm{ab}\end{array}$ & $\begin{array}{c}1114 \\
\mathrm{a}\end{array}$ & $\begin{array}{c}525.0 \\
\text { b }\end{array}$ & $\begin{array}{c}564.8 \\
\text { a-d }\end{array}$ & $\begin{array}{c}537.8 \\
\text { a-e }\end{array}$ & $\begin{array}{c}472.5 \\
b-f\end{array}$ & $\begin{array}{c}342.0 \\
\mathrm{ab}\end{array}$ & $\begin{array}{c}281.5 \\
\mathrm{c}-\mathrm{g}\end{array}$ & $\begin{array}{c}329.2 \\
\text { b-e }\end{array}$ & $\begin{array}{c}415.4 \\
\text { abc }\end{array}$ & $1 \ldots$ \\
\hline $\begin{array}{c}830.9 \\
\text { b }\end{array}$ & $\begin{array}{c}745.8 \\
\text { d-h }\end{array}$ & $\begin{array}{c}858.4 \\
\text { c-f }\end{array}$ & $\begin{array}{c}888.6 \\
\text { b-e }\end{array}$ & $\begin{array}{c}677.0 \\
\mathrm{a}\end{array}$ & $\begin{array}{c}600.0 \\
\text { abc }\end{array}$ & $\begin{array}{c}667.7 \\
\mathrm{ab}\end{array}$ & $\begin{array}{c}763.3 \\
\mathrm{a}\end{array}$ & $\begin{array}{c}393.3 \\
\mathrm{a}\end{array}$ & $\begin{array}{c}321.8 \\
\text { b-e }\end{array}$ & $\begin{array}{c}418.4 \\
\mathrm{Abc}\end{array}$ & $\begin{array}{c}439.8 \\
\mathrm{ab}\end{array}$ & $10 \ldots$ \\
\hline $\begin{array}{c}681.2 \\
c\end{array}$ & $\begin{array}{c}617.2 \\
\text { g-k }\end{array}$ & $\begin{array}{c}732.0 \\
\text { d-h }\end{array}$ & $\begin{array}{c}694.5 \\
\text { e-h }\end{array}$ & $\begin{array}{c}499.5 \\
b\end{array}$ & $\begin{array}{c}505.4 \\
\text { a-e }\end{array}$ & $\begin{array}{c}454.5 \\
\text { b-f }\end{array}$ & $\begin{array}{c}538.9 \\
\text { a-e }\end{array}$ & $\begin{array}{c}393.4 \\
\mathrm{a}\end{array}$ & $\begin{array}{c}285.4 \\
\mathrm{c}-\mathrm{g}\end{array}$ & $\begin{array}{c}390.4 \\
\text { a-d }\end{array}$ & $\begin{array}{c}504.5 \\
a\end{array}$ & $r \ldots$ \\
\hline $\begin{array}{c}561.3 \\
d\end{array}$ & $\begin{array}{c}585.6 \\
\text { g-1 }\end{array}$ & $\begin{array}{c}524.0 \\
\text { h-m }\end{array}$ & $\begin{array}{c}574.2 \\
\text { g-1 }\end{array}$ & $\begin{array}{c}445.9 \\
\text { bc }\end{array}$ & $\begin{array}{c}428.8 \\
\text { b-f }\end{array}$ & $\begin{array}{c}414.5 \\
b-f\end{array}$ & $\begin{array}{c}494.4 \\
\text { b-f }\end{array}$ & $\begin{array}{c}277.8 \\
\text { bc }\end{array}$ & $\begin{array}{c}224.3 \\
\text { e-h }\end{array}$ & $\begin{array}{c}279.6 \\
\mathrm{c}-\mathrm{g}\end{array}$ & $\begin{array}{c}329.6 \\
b-c\end{array}$ & ro.. \\
\hline $\begin{array}{c}445.2 \\
\mathrm{e}\end{array}$ & $\begin{array}{c}444.6 \\
\text { j-h }\end{array}$ & $\begin{array}{c}425.3 \\
\mathrm{k}-\mathrm{n}\end{array}$ & $\begin{array}{c}465.8 \\
\text { i-n }\end{array}$ & $\begin{array}{c}376.2 \\
\text { bcd }\end{array}$ & $\begin{array}{c}321.2 \\
c-f\end{array}$ & $\begin{array}{c}378.5 \\
c-f\end{array}$ & $\begin{array}{c}428.8 \\
b-f\end{array}$ & $\begin{array}{c}188.1 \\
\text { de }\end{array}$ & $\begin{array}{c}144.7 \\
\text { ghi }\end{array}$ & $\begin{array}{c}173.9 \\
\mathrm{f}-\mathrm{i}\end{array}$ & $\begin{array}{c}245.7 \\
\text { e-h }\end{array}$ & r... \\
\hline $\begin{array}{c}394.6 \\
\mathrm{e}\end{array}$ & $\begin{array}{c}404.6 \\
k-n\end{array}$ & $\begin{array}{c}349.9 \\
\mathrm{mn}\end{array}$ & $\begin{array}{c}429.8 \\
k-n\end{array}$ & $\begin{array}{c}346.8 \\
\mathrm{~cd}\end{array}$ & $\begin{array}{c}286.7 \\
\text { def }\end{array}$ & $\begin{array}{c}324.4 \\
c-f\end{array}$ & $\begin{array}{c}429.3 \\
b-f\end{array}$ & $\begin{array}{c}152.1 \\
\text { ef }\end{array}$ & $\begin{array}{c}106.0 \\
\text { hi }\end{array}$ & $\begin{array}{c}144.2 \\
\text { Ghi }\end{array}$ & $\begin{array}{c}206.1 \\
\text { e-i }\end{array}$ & ro.. \\
\hline $\begin{array}{c}334.5 \\
\mathrm{e}\end{array}$ & $\begin{array}{c}295.1 \\
\mathrm{n}\end{array}$ & $\begin{array}{c}315.7 \\
\mathrm{mn}\end{array}$ & $\begin{array}{l}392.7 \\
\text { Lmn }\end{array}$ & $\begin{array}{c}279.9 \\
\mathrm{~d}\end{array}$ & $\begin{array}{c}220.7 \\
\mathrm{f}\end{array}$ & $\begin{array}{c}273.5 \\
\text { ef }\end{array}$ & $\begin{array}{c}345.6 \\
c-f\end{array}$ & $\begin{array}{c}112.8 \\
\mathrm{f}\end{array}$ & $\begin{array}{c}\text { 78. } 50 \\
\mathrm{i}\end{array}$ & $\begin{array}{c}107.9 \\
\mathrm{Hi}\end{array}$ & $\begin{array}{c}151.9 \\
\text { ghi }\end{array}$ & $\varepsilon \ldots$ \\
\hline & $\begin{array}{c}609.6 \\
b\end{array}$ & $\begin{array}{c}650.9 \\
\mathrm{ab}\end{array}$ & $\begin{array}{c}691.2 \\
\mathrm{a}\end{array}$ & & $\begin{array}{c}413.1 \\
\mathrm{a}\end{array}$ & $\begin{array}{c}438.8 \\
\mathrm{a}\end{array}$ & $\begin{array}{c}480.6 \\
\mathrm{a}\end{array}$ & & $\begin{array}{c}211.4 \\
\mathrm{c}\end{array}$ & $\begin{array}{c}260.7 \\
\text { B }\end{array}$ & $\begin{array}{c}314.9 \\
\mathrm{a}\end{array}$ & للرش المعدل \\
\hline
\end{tabular}

المعدلات التي تشترك بنفس الأحرف لكل عمود لا تختلف معنويا حسب اختبار دنكن متعدد الحدود عند مستوى احتمال % 


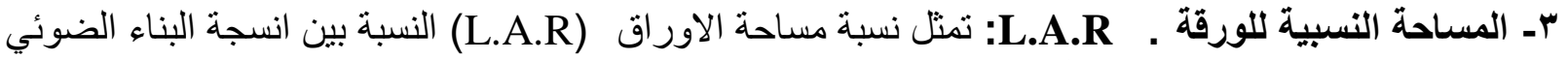

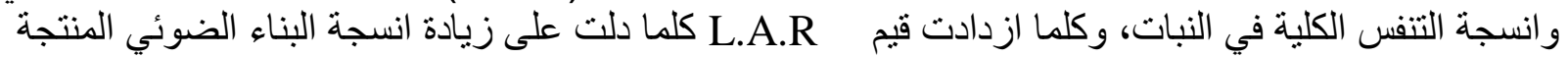

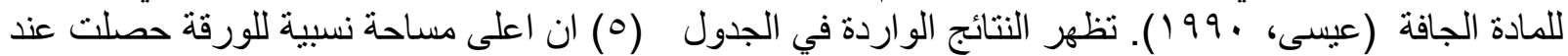

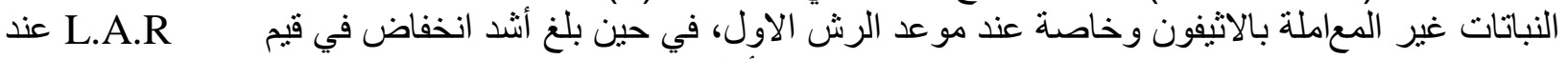

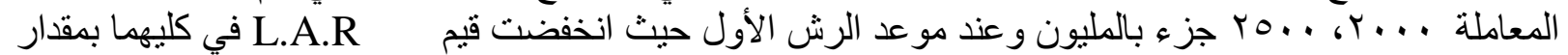

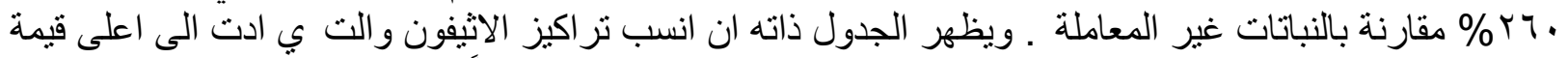

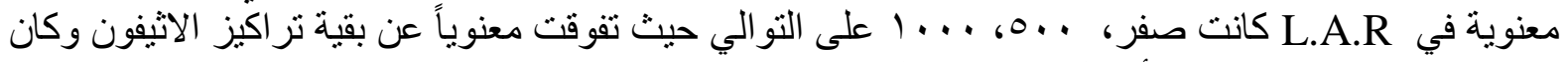

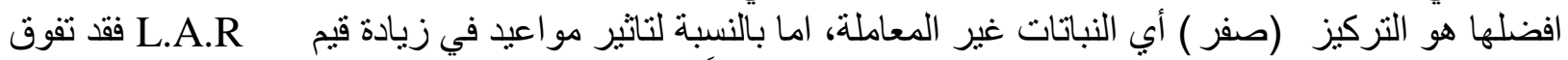

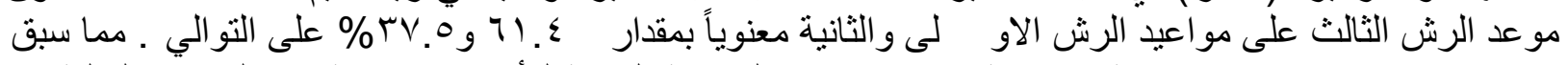

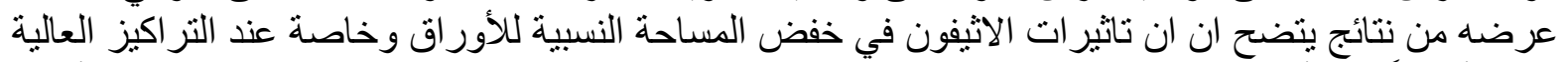

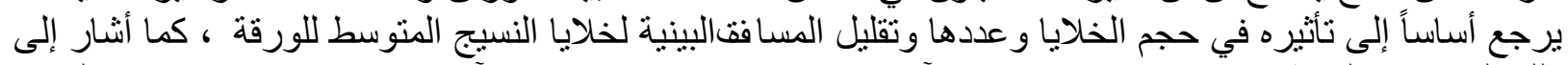

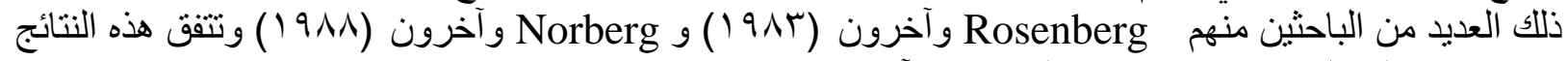

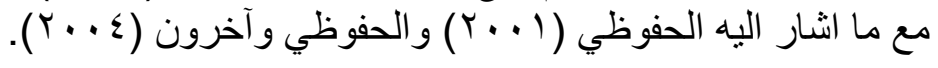

الجدول (0) نأثثرات تر اكيز ومو اعيد رش الاثيفون في المساحة النسبية للورقة L.A.R دسمَ /غم /اسنوع

\begin{tabular}{|c|c|c|c|c|c|c|c|c|c|c|}
\hline للمو اعيد & $\{\ldots$ & ro.. & $r \ldots$ & ro.. & r... & $10 \ldots$ & $1 \ldots$ & $0 .$. & صفر & التركيز ppm الرش \\
\hline $\begin{array}{c}0.018 \\
\mathrm{~B}\end{array}$ & $\begin{array}{c}0.014 \\
\text { fg }\end{array}$ & $\begin{array}{c}0.012 \\
\mathrm{~g}\end{array}$ & $\begin{array}{c}0.011 \\
\mathrm{~g}\end{array}$ & $\begin{array}{c}0.010 \\
\mathrm{~g}\end{array}$ & $\begin{array}{c}0.010 \\
\mathrm{~g}\end{array}$ & $\begin{array}{c}0.014 \\
f-g\end{array}$ & $\begin{array}{c}0.026 \\
\text { a-f }\end{array}$ & $\begin{array}{c}0.030 \\
\text { a-d }\end{array}$ & $\begin{aligned} \because & \cdot 4 T \\
& \mathrm{a}\end{aligned}$ & الاول \\
\hline $\begin{array}{c}0.021 \\
\mathrm{~B}\end{array}$ & $\begin{array}{c}0.019 \\
\mathrm{c}-\mathrm{g}\end{array}$ & $\begin{array}{c}0.016 \\
\text { efg }\end{array}$ & $\begin{array}{c}0.016 \\
\text { efg }\end{array}$ & $\begin{array}{c}0.012 \\
\mathrm{~g}\end{array}$ & $\begin{array}{c}0.015 \\
\mathrm{fg}\end{array}$ & $\begin{array}{c}0.018 \\
\mathrm{~d}-\mathrm{g}\end{array}$ & $\begin{array}{c}0.034 \\
\mathrm{a}\end{array}$ & $\begin{array}{c}0.033 \\
\mathrm{ab}\end{array}$ & $\begin{array}{c}0.029 \\
\text { a-e }\end{array}$ & الثاني \\
\hline $\begin{array}{c}0.029 \\
\mathrm{~A}\end{array}$ & $\begin{array}{c}0.032 \\
\mathrm{abc}\end{array}$ & $\begin{array}{c}0.034 \\
\mathrm{a}\end{array}$ & $\begin{array}{c}0.029 \\
\text { a-e }\end{array}$ & $\begin{array}{c}0.026 \\
\text { a-f }\end{array}$ & $\begin{array}{c}0.019 \\
\mathrm{c}-\mathrm{g}\end{array}$ & $\begin{array}{c}0.020 \\
b-g\end{array}$ & $\begin{array}{c}0.035 \\
\mathrm{a}\end{array}$ & $\begin{array}{c}0.035 \\
\mathrm{a}\end{array}$ & $\begin{array}{c}0.035 \\
\mathrm{a}\end{array}$ & الثالث \\
\hline & $\begin{array}{c}0.022 \\
\mathrm{~b}\end{array}$ & $\begin{array}{c}0.020 \\
\mathrm{~b}\end{array}$ & $\begin{array}{c}0.018 \\
\mathrm{~b}\end{array}$ & $\begin{array}{c}0.016 \\
b\end{array}$ & $\begin{array}{c}0.014 \\
b\end{array}$ & $\begin{array}{c}0.017 \\
b\end{array}$ & $\begin{array}{c}0.032 \\
\mathrm{a}\end{array}$ & $\begin{array}{c}0.032 \\
\mathrm{a}\end{array}$ & $\begin{array}{c}0.033 \\
\mathrm{a}\end{array}$ & للتركيز \\
\hline
\end{tabular}

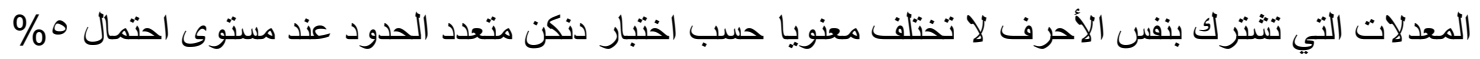

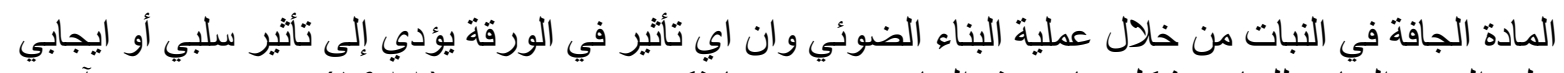

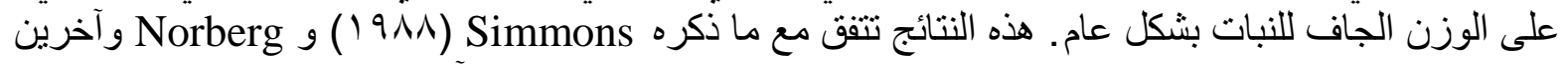

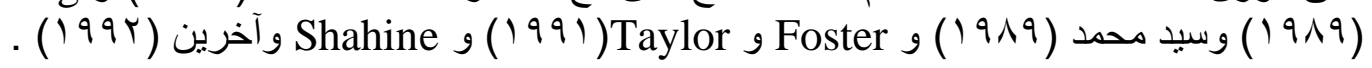

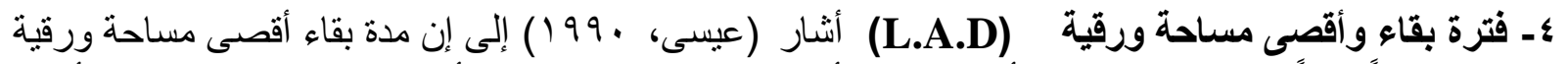

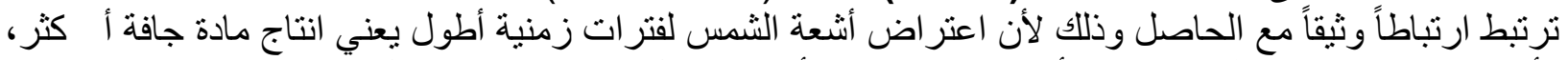

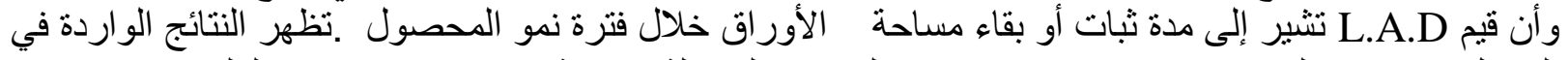

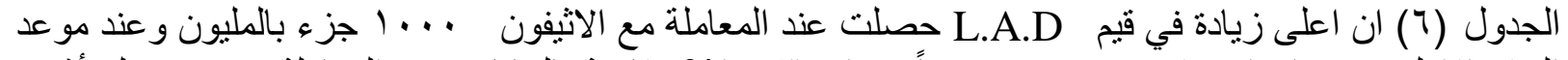

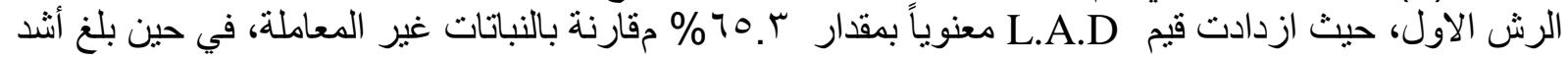

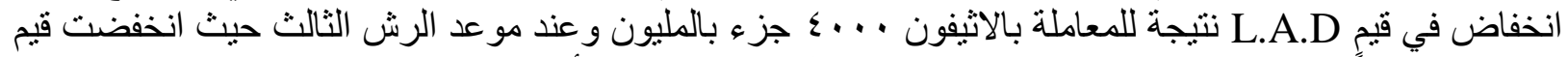

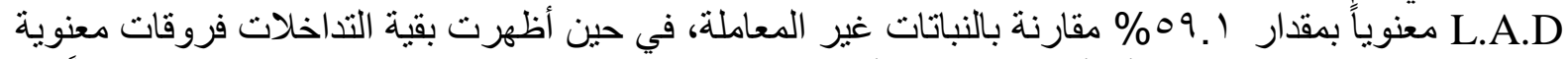

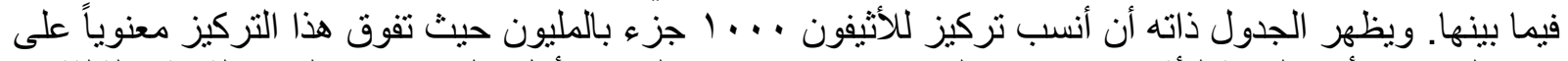

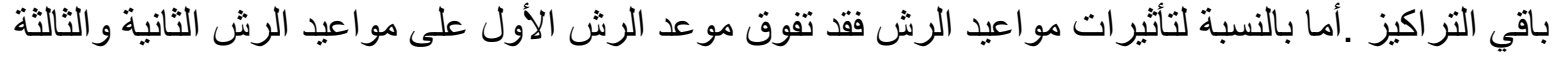




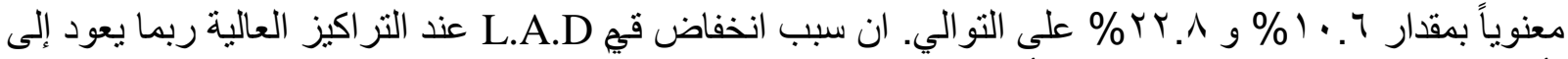

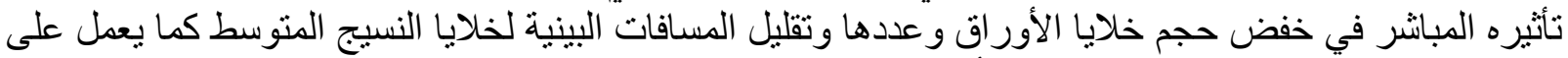

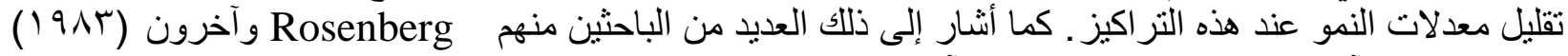

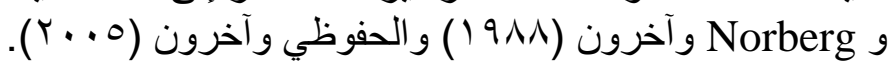

الجدول (T) تأثير تر اكيز ومو اعبد رش الاثيفون في فترة بقاء أقصى مساحة ورقية L.A.D دسمَ / اسبوع

\begin{tabular}{|c|c|c|c|c|c|c|c|c|c|c|}
\hline اللمواعد & $\varepsilon \ldots$ & ro... & $r \ldots$ & Yo.. & r... & $10 \ldots$ & $1 \ldots$ & $0 .$. & صفر & موعد الرش ppm \\
\hline $\begin{array}{c}40.32 \\
\mathrm{a}\end{array}$ & $\begin{array}{c}21.78 \\
j-m\end{array}$ & $\begin{array}{c}25.42 \\
\mathrm{i}-\mathrm{m}\end{array}$ & $\begin{array}{c}28.45 \\
\text { h-1 }\end{array}$ & $\begin{array}{c}36.14 \\
\mathrm{f}-\mathrm{i}\end{array}$ & $\begin{array}{c}47.95 \\
\text { b-f }\end{array}$ & $\begin{array}{c}53.13 \\
\mathrm{abc}\end{array}$ & $\begin{array}{c}61.17 \\
\mathrm{a}\end{array}$ & $\begin{array}{c}51.87 \\
\text { a-d }\end{array}$ & $\begin{array}{c}r v_{.}+1 \\
\mathrm{e}-\mathrm{i}\end{array}$ & الاول \\
\hline $\begin{array}{c}36.45 \\
\mathrm{~b}\end{array}$ & $\begin{array}{c}16.94 \\
\operatorname{lm}\end{array}$ & $\begin{array}{c}19.75 \\
\operatorname{lm}\end{array}$ & $\begin{array}{c}23.96 \\
\mathrm{j}-\mathrm{m}\end{array}$ & $\begin{array}{c}32.14 \\
\text { g-k }\end{array}$ & $\begin{array}{c}44.89 \\
\text { b-f }\end{array}$ & $\begin{array}{c}51.06 \\
\text { a-d }\end{array}$ & $\begin{array}{c}56.40 \\
\mathrm{ab}\end{array}$ & $\begin{array}{c}47.00 \\
b-f\end{array}$ & $\begin{array}{c}35.96 \\
f-i\end{array}$ & الثاني \\
\hline $\begin{array}{c}32.83 \\
\mathrm{c}\end{array}$ & $\begin{array}{c}14.95 \\
\mathrm{~m}\end{array}$ & $\begin{array}{c}20.35 \\
\text { klm }\end{array}$ & $\begin{array}{c}23.57 \\
\mathrm{j}-\mathrm{m}\end{array}$ & $\begin{array}{c}32.39 \\
\mathrm{~g}-\mathrm{j}\end{array}$ & $\begin{array}{c}36.1 \\
\mathrm{f}-\mathrm{i}\end{array}$ & $\begin{array}{c}42.70 \\
\mathrm{c}-\mathrm{g}\end{array}$ & $\begin{array}{c}48.51 \\
b-e\end{array}$ & $\begin{array}{c}40.35 \\
\text { d-h }\end{array}$ & $\begin{array}{c}36.56 \\
\mathrm{f}-\mathrm{i}\end{array}$ & الثالث \\
\hline & $\begin{array}{c}17.89 \\
\mathrm{e}\end{array}$ & $\begin{array}{c}21.63 \\
\text { de }\end{array}$ & $\begin{array}{c}25.33 \\
\mathrm{~d}\end{array}$ & $\begin{array}{c}33.56 \\
\mathrm{c}\end{array}$ & $\begin{array}{c}42.98 \\
\mathrm{~b}\end{array}$ & $\begin{array}{c}48.96 \\
\text { b }\end{array}$ & $\begin{array}{c}55.36 \\
\mathrm{a}\end{array}$ & $\begin{array}{c}46.411 \\
\text { B }\end{array}$ & $\begin{array}{c}36.51 \\
\mathrm{c}\end{array}$ & اللتركيز \\
\hline
\end{tabular}

المعدلات التي تشترك بنفس الاحرف لا تختلف معنويا حسب اختبار دنكن متعدد الحدود عند مستوى احتمال 0\%

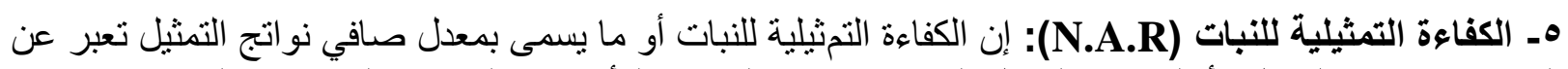

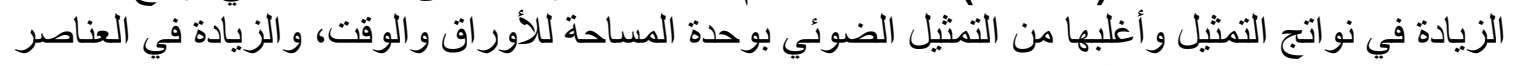

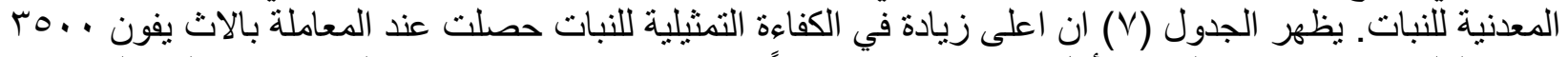

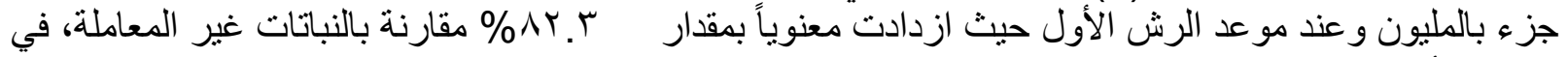

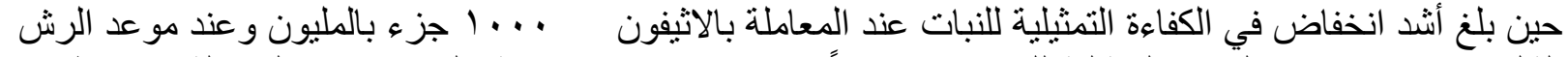

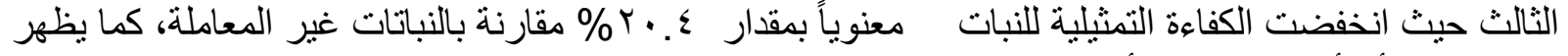

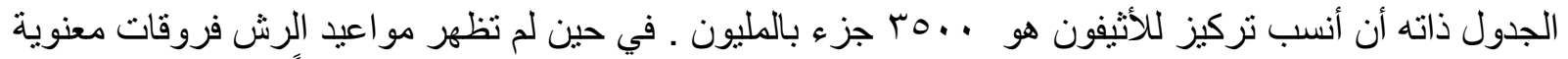

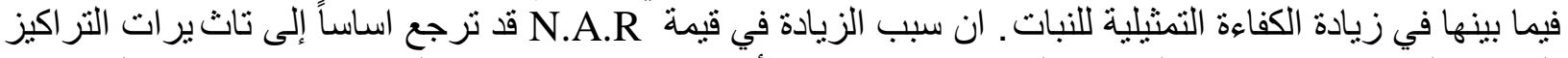

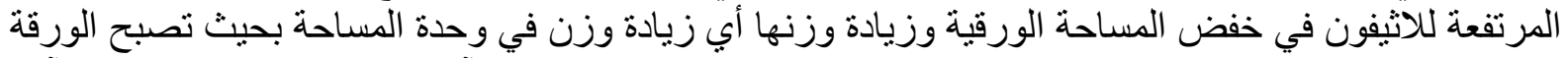

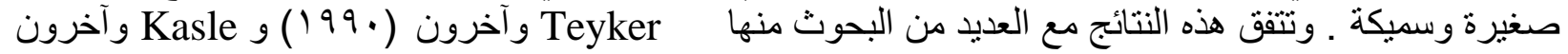

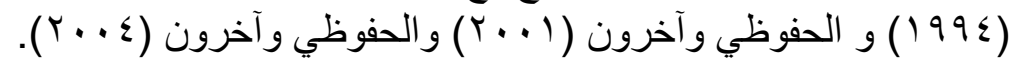

الجدول (V) تأثير تر اكيز ومو اعيد رش الاثيفون في الكفاءة التمثلية للنبات N.A.R غم/دم//سبوع

\begin{tabular}{|c|c|c|c|c|c|c|c|c|c|c|}
\hline للمواع المعل & $\varepsilon \ldots$ & ro.. & $r \ldots$ & ro.. & r... & $10 \ldots$ & $1 \ldots$ & $0 .$. & صفر & موعد الرش ppm \\
\hline $\begin{array}{c}\cdot r Y Y \\
a\end{array}$ & $\begin{array}{c}0.263 \\
\text { abc }\end{array}$ & $\begin{array}{c}0.310 \\
\mathrm{a}\end{array}$ & $\begin{array}{c}0.276 \\
a b\end{array}$ & $\begin{array}{c}0.250 \\
\text { a-d }\end{array}$ & $\begin{array}{c}0.206 \\
\text { bcd }\end{array}$ & $\begin{array}{c}0.233 \\
\text { a-d }\end{array}$ & $\begin{array}{c}0.173 \\
\mathrm{~cd}\end{array}$ & $\begin{array}{c}0.183 \\
\text { Bcd }\end{array}$ & $\begin{array}{c}60.17 \\
\mathrm{~cd}\end{array}$ & الاول \\
\hline $\begin{array}{c}0.219 \\
\mathrm{a}\end{array}$ & $\begin{array}{c}0.273 \\
a b\end{array}$ & $\begin{array}{c}0.276 \\
a b\end{array}$ & $\begin{array}{l}\cdot r 4 . \\
a b c\end{array}$ & $\begin{array}{c}0.213 \\
\text { bcd }\end{array}$ & $\begin{array}{c}0.183 \\
\text { bcd }\end{array}$ & $\begin{array}{c}0.210 \\
\text { bcd }\end{array}$ & $\begin{array}{c}0.190 \\
\text { bgd }\end{array}$ & $\begin{array}{c}0.173 \\
\mathrm{Cd} \\
\end{array}$ & $\begin{array}{l}\because 194 \\
\text { bcd }\end{array}$ & الثاني \\
\hline
\end{tabular}




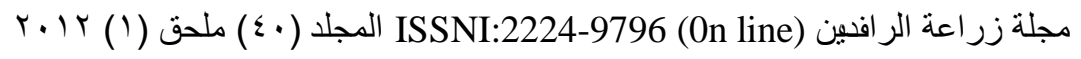
ISSN:1815-316X (Print)

\begin{tabular}{|c|c|c|c|c|c|c|c|c|c|c|}
\hline $\begin{array}{c}0.207 \\
\mathrm{a}\end{array}$ & $\begin{array}{c}0.233 \\
\text { a-d }\end{array}$ & $\begin{array}{c}0.233 \\
\text { a-d }\end{array}$ & $\begin{array}{c}0.220 \\
\text { a-d }\end{array}$ & $\begin{array}{c}0.186 \\
\text { bcd }\end{array}$ & $\begin{array}{c}0.210 \\
\text { bcd }\end{array}$ & $\begin{array}{c}0.216 \\
\text { a-d }\end{array}$ & $\begin{array}{c}0.156 \\
\mathrm{~d}\end{array}$ & $\begin{array}{c}0.193 \\
\text { Bcd }\end{array}$ & $\begin{array}{c}0.196 \\
\text { bcd }\end{array}$ & الثالث \\
\hline & $\begin{array}{c}0.256 \\
\text { ab }\end{array}$ & $\begin{array}{c}0.273 \\
\mathrm{a}\end{array}$ & $\begin{array}{c}0.252 \\
a b\end{array}$ & $\begin{array}{c}0.216 \\
\text { bc }\end{array}$ & 0.200 & $\begin{array}{c}0.220 \\
\text { bc }\end{array}$ & $\begin{array}{c}0.173 \\
\mathrm{c}\end{array}$ & $\begin{array}{c}0.183 \\
\mathrm{C}\end{array}$ & $\begin{array}{c}0.186 \\
\mathrm{c}\end{array}$ & للتركيزل \\
\hline
\end{tabular}

المعدلات التي تثترك بنفس الاحرف لا تختلف معنويا حسب اختبار دنكن متعدد الحدود عند مستوى احتمال 0\%

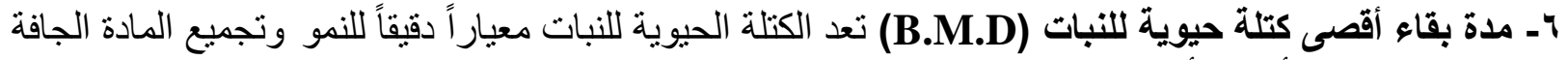

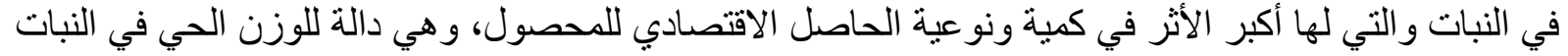

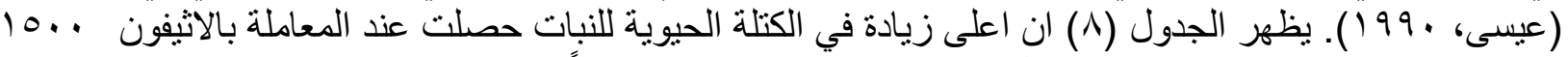

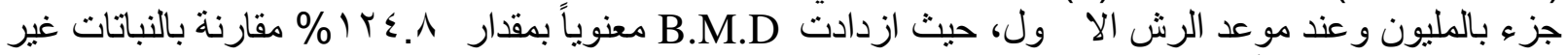

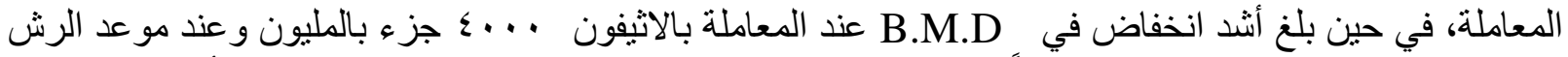

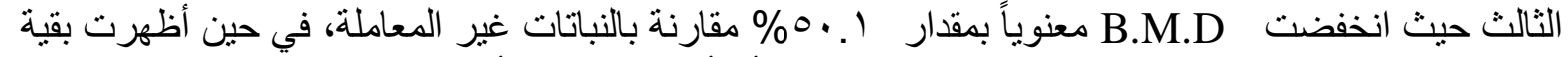

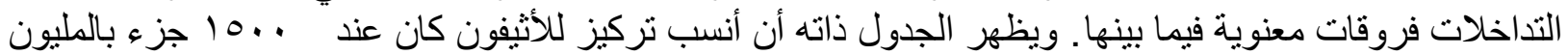

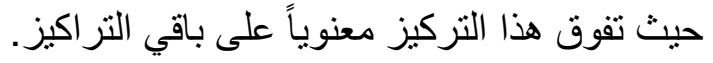

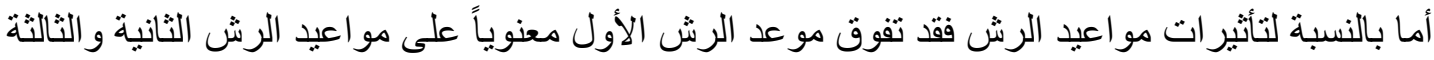

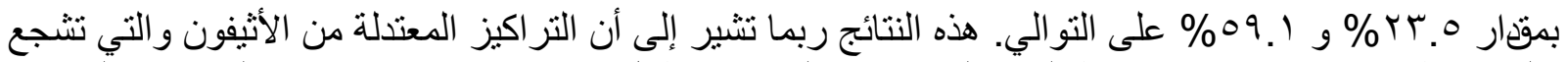

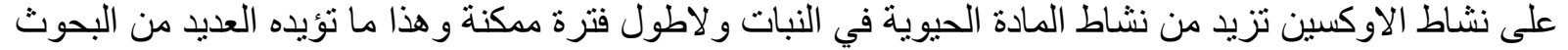

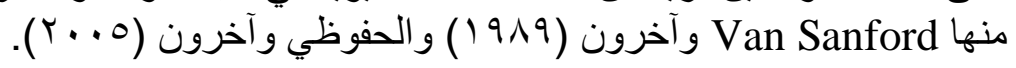

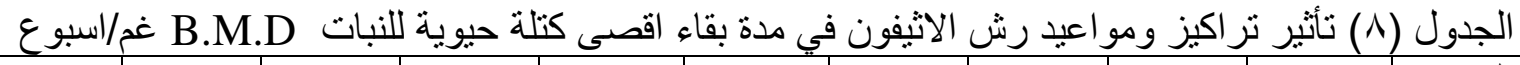

\begin{tabular}{|c|c|c|c|c|c|c|c|c|c|c|}
\hline للمواع المعد & $\varepsilon \ldots$ & ro.. & $r \ldots$ & ro.. & r... & $10 \ldots$ & $1 \ldots$ & $0 \ldots$ & صفر & الترشئ \\
\hline $\begin{array}{c}116.0 \\
\mathrm{a}\end{array}$ & $\begin{array}{c}72.24 \\
\text { i-1 }\end{array}$ & $\begin{array}{c}117.2 \\
\text { b-e }\end{array}$ & $\begin{array}{c}105.7 \\
\text { def }\end{array}$ & $\begin{array}{c}120.5 \\
\text { bcd }\end{array}$ & $\begin{array}{c}138.9 \\
\text { b }\end{array}$ & $\begin{array}{c}167.9 \\
\mathrm{a}\end{array}$ & $\begin{array}{c}135.9 \\
\text { bc }\end{array}$ & $\begin{array}{c}111.2 \\
\text { De }\end{array}$ & $\begin{array}{c}v \leqslant .79 \\
h-k\end{array}$ & الاول \\
\hline $\begin{array}{c}94.02 \\
\mathrm{~b}\end{array}$ & $\begin{array}{c}58.78 \\
\text { klm }\end{array}$ & $\begin{array}{c}69.05 \\
\mathrm{jkl}\end{array}$ & $\begin{array}{c}78.70 \\
\text { g-k }\end{array}$ & $\begin{array}{c}94.72 \\
\text { e-i }\end{array}$ & $\begin{array}{c}112.0 \\
\mathrm{de}\end{array}$ & $\begin{array}{c}137.8 \\
\text { bc }\end{array}$ & $\begin{array}{c}114.2 \\
\text { cde }\end{array}$ & $\begin{array}{c}101.3 \\
\mathrm{~d}-\mathrm{g}\end{array}$ & $\begin{array}{c}79.41 \\
\text { g-k }\end{array}$ & الثاني \\
\hline $\begin{array}{c}76.32 \\
\mathrm{c}\end{array}$ & $\begin{array}{c}38.62 \\
\mathrm{~m}\end{array}$ & $\begin{array}{c}49.49 \\
1 \mathrm{~m}\end{array}$ & $\begin{array}{c}58.30 \\
\text { klm }\end{array}$ & $\begin{array}{c}V T . r \varepsilon \\
i-1\end{array}$ & $\begin{array}{c}q 4.1 \cdot \\
e-j\end{array}$ & $\begin{array}{c}111.5 \\
\text { de }\end{array}$ & $\begin{array}{c}98.4 \\
\text { d-h }\end{array}$ & $\begin{array}{c}86.70 \\
\mathrm{f}-\mathrm{j} \\
\end{array}$ & $\begin{array}{c}77.38 \\
\text { g-k }\end{array}$ & الثالث \\
\hline & $\begin{array}{c}56.55 \\
\mathrm{e}\end{array}$ & $\begin{array}{c}78.58 \\
\mathrm{~d}\end{array}$ & $\begin{array}{c}80.92 \\
\mathrm{~d}\end{array}$ & $\begin{array}{c}96.20 \\
\mathrm{c}\end{array}$ & $\begin{array}{c}114.2 \\
\mathrm{~b}\end{array}$ & $\begin{array}{c}139.1 \\
\mathrm{a}\end{array}$ & $\begin{array}{c}116.2 \\
\mathrm{~b}\end{array}$ & $\begin{array}{c}94.77 \\
\text { C }\end{array}$ & $\begin{array}{c}77.16 \\
\mathrm{~d}\end{array}$ & اللتركيز \\
\hline
\end{tabular}

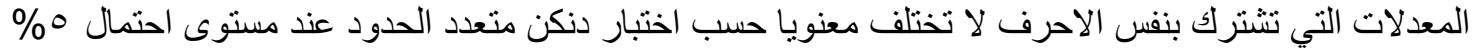

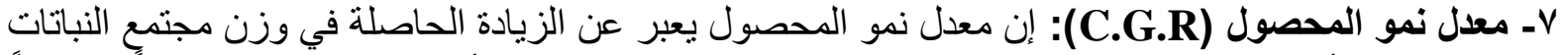

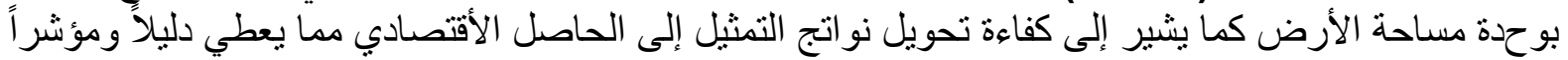

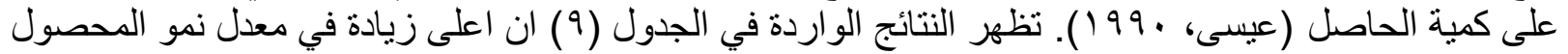

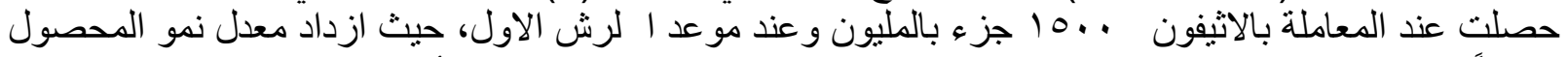

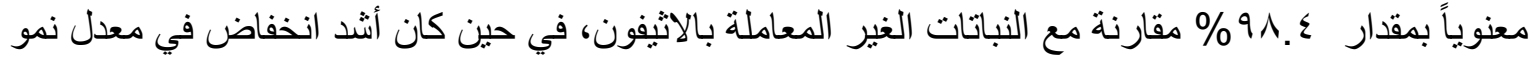

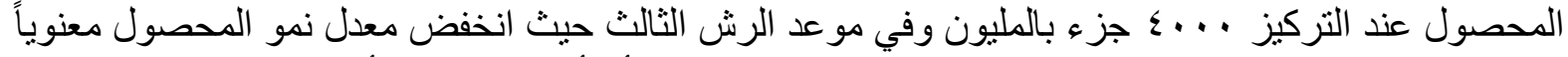

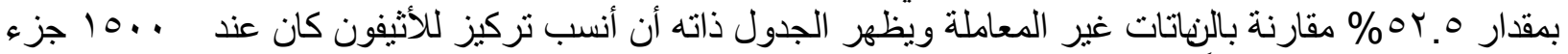

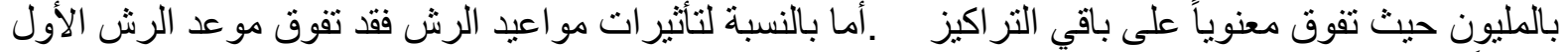

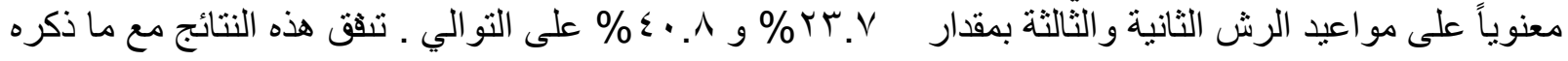




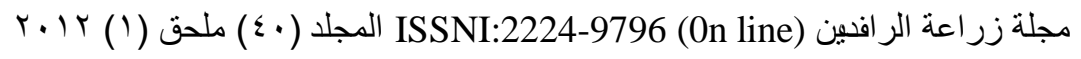
ISSN:1815-316X (Print)

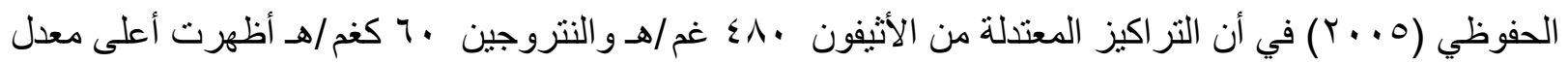

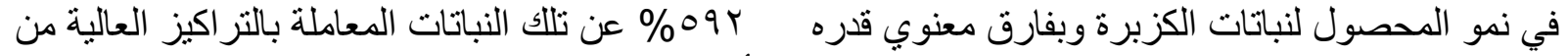

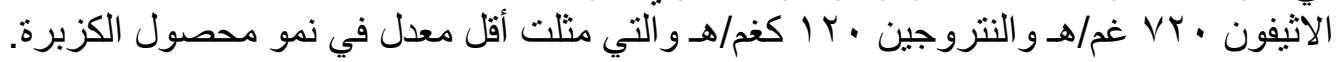

\begin{tabular}{|c|c|c|c|c|c|c|c|c|c|c|}
\hline اللمواع & $\varepsilon \ldots$ & ro.. & $r \ldots$. & ro.. & r... & $10 \ldots$ & $1 \ldots$ & $0 .$. & صفر & الترش \\
\hline $\begin{array}{c}5.520 \\
\mathrm{a}\end{array}$ & $\begin{array}{c}3.464 \\
\text { i-n }\end{array}$ & $\begin{array}{c}4.261 \\
\mathrm{f}-1\end{array}$ & $\begin{array}{c}5.99 \\
\text { b-e }\end{array}$ & $\begin{array}{c}5.623 \\
\text { b-f }\end{array}$ & $\begin{array}{c}6.422 \\
\text { bcd }\end{array}$ & $\begin{array}{c}7.972 \\
\mathrm{a}\end{array}$ & $\begin{array}{c}6.620 \\
\text { abc }\end{array}$ & $\begin{array}{c}5.199 \\
\text { b-h }\end{array}$ & $\begin{array}{c}4.017 \\
\text { g-m }\end{array}$ & الاول \\
\hline $\begin{array}{c}4.464 \\
\text { b }\end{array}$ & $\begin{array}{c}2.738 \\
\operatorname{lmn}\end{array}$ & $\begin{array}{c}3.295 \\
\text { j-n }\end{array}$ & $\begin{array}{c}3.693 \\
\text { h-m }\end{array}$ & $\begin{array}{c}4.325 \\
\mathrm{f}-\mathrm{k}\end{array}$ & $\begin{array}{c}5.103 \\
\text { c-h }\end{array}$ & $\begin{array}{c}6.670 \\
a b\end{array}$ & $\begin{array}{c}5.427 \\
\text { b-g }\end{array}$ & $\begin{array}{c}4.744 \\
\mathrm{e}-\mathrm{j}\end{array}$ & $\begin{array}{c}4.180 \\
\mathrm{f}-\mathrm{m}\end{array}$ & الثاني \\
\hline $\begin{array}{c}3.920 \\
\mathrm{c}\end{array}$ & $\begin{array}{c}2.006 \\
n\end{array}$ & $\begin{array}{c}2.637 \\
\mathrm{mn}\end{array}$ & $\begin{array}{c}2.990 \\
\text { k-n }\end{array}$ & $\begin{array}{c}3.715 \\
\text { h-m }\end{array}$ & $\begin{array}{c}4.608 \\
e-j\end{array}$ & $\begin{array}{c}5.562 \\
\text { b-g }\end{array}$ & $\begin{array}{c}4.994 \\
\text { d-i }\end{array}$ & $\begin{array}{c}4.547 \\
\text { e-k }\end{array}$ & $\begin{array}{c}4.223 \\
\mathrm{f}-1\end{array}$ & الثالث \\
\hline & $\begin{array}{c}2.736 \\
\mathrm{f}\end{array}$ & $\begin{array}{c}3.398 \\
\text { ef }\end{array}$ & $\begin{array}{c}4.260 \\
\mathrm{~d}\end{array}$ & $\begin{array}{c}4.554 \\
\mathrm{~d}\end{array}$ & $\begin{array}{c}5.378 \\
\text { bc }\end{array}$ & $\begin{array}{c}6.735 \\
\mathrm{a}\end{array}$ & $\begin{array}{c}5.680 \\
\text { b }\end{array}$ & $\begin{array}{c}4.830 \\
\mathrm{Cd}\end{array}$ & $\begin{array}{c}4.140 \\
\text { de }\end{array}$ & للتركيز \\
\hline
\end{tabular}

1 - تركيز البوتاسيوم في البذور : يظهر الجدول (· · () أن أعلى زيادة في تركيز البوتاسيوم في بذور الحلبة

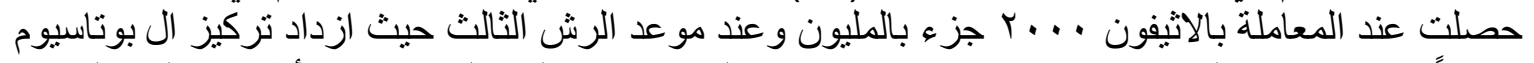

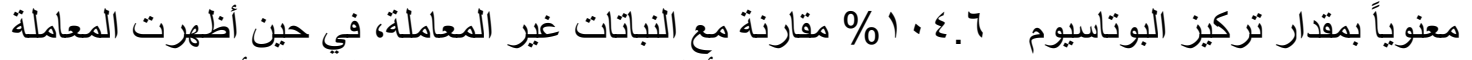

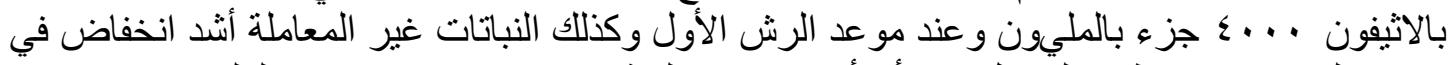

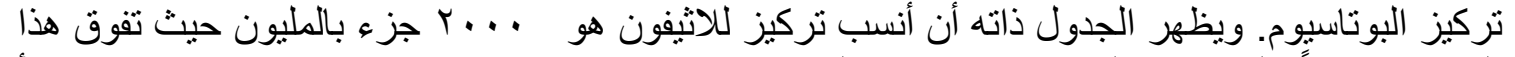

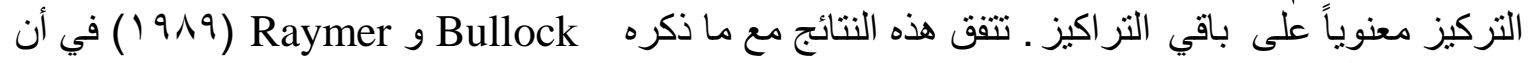

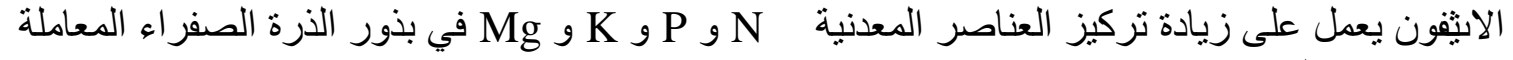

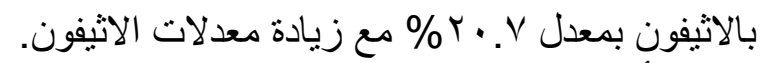
الجدول (· • ) تأثير نر اكيز ومو اعيد رش الاثيفون في صيفة تركيز البوتاسيوم ملغ م/غم وزن جاف للبذور

\begin{tabular}{|c|c|c|c|c|c|c|c|c|c|c|}
\hline للمواع المعدل & $\{\ldots$ & ro.. & r... & ro.. & $r \ldots$ & $10 .$. & $1 \ldots$ & $0 .$. & صفر & 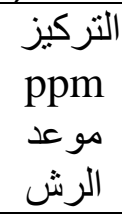 \\
\hline $\begin{array}{c}5.488 \\
\mathrm{c}\end{array}$ & $\begin{array}{c}4.364 \\
\mathrm{o}\end{array}$ & $\begin{array}{c}5.356 \\
\text { k-n }\end{array}$ & $\begin{array}{c}5.555 \\
\mathrm{j}-\mathrm{m}\end{array}$ & $\begin{array}{c}5.753 \\
\mathrm{i}-\mathrm{m}\end{array}$ & $\begin{array}{c}5.356 \\
\text { k-n }\end{array}$ & $\begin{array}{c}5.959 \\
\text { mno }\end{array}$ & $\begin{array}{c}6.150 \\
\text { g-k }\end{array}$ & $\begin{array}{c}7.340 \\
\text { b-e }\end{array}$ & $\begin{array}{c}\varepsilon .07 r \\
\text { no }\end{array}$ & الاول \\
\hline $\begin{array}{c}6.260 \\
b\end{array}$ & $\begin{array}{c}5.158 \\
1-0\end{array}$ & $\begin{array}{c}5.952 \\
\text { h-1 }\end{array}$ & $\begin{array}{c}6.547 \\
\text { e-i }\end{array}$ & $\begin{array}{c}6.348 \\
f-j\end{array}$ & $\begin{array}{c}7.539 \\
\text { bcd }\end{array}$ & $\begin{array}{c}6.547 \\
\mathrm{e}-\mathrm{i}\end{array}$ & $\begin{array}{c}7.142 \\
\text { b-f }\end{array}$ & $\begin{array}{c}6.745 \\
\text { d-h }\end{array}$ & $\begin{array}{c}4.364 \\
\mathrm{o}\end{array}$ & الثاني \\
\hline $\begin{array}{c}7.054 \\
\mathrm{a}\end{array}$ & $\begin{array}{c}6.150 \\
\text { g-k }\end{array}$ & $\begin{array}{c}6.943 \\
\mathrm{c}-\mathrm{g}\end{array}$ & $\begin{array}{c}7.737 \\
\text { bc }\end{array}$ & $\begin{array}{c}7.936 \\
b\end{array}$ & $\begin{array}{c}8.928 \\
\text { a }\end{array}$ & $\begin{array}{c}7.737 \\
\text { bc }\end{array}$ & $\begin{array}{c}6.745 \\
\text { d-h }\end{array}$ & $\begin{array}{c}6.943 \\
\mathrm{c}-\mathrm{g}\end{array}$ & $\begin{array}{c}4.364 \\
\mathrm{o}\end{array}$ & الثالث \\
\hline & $\begin{array}{c}5.224 \\
\mathrm{e}\end{array}$ & $\begin{array}{c}6.084 \\
\mathrm{~d}\end{array}$ & $\begin{array}{c}6.613 \\
\text { bc }\end{array}$ & $\begin{array}{c}6.679 \\
\text { bc }\end{array}$ & $\begin{array}{c}7.274 \\
\mathrm{a}\end{array}$ & $\begin{array}{c}6.414 \\
\mathrm{~cd}\end{array}$ & $\begin{array}{c}6.679 \\
\text { bc }\end{array}$ & $\begin{array}{c}7.00 \\
\mathrm{ab}\end{array}$ & $\begin{array}{c}4.430 \\
\text { f }\end{array}$ & للتركيزل \\
\hline
\end{tabular}

المعدلات التي تثترك بنفس الاحرف لا تختلف معنويا حسب اختبار دنكن متعدد الحدود عند مستوى احتمال 0\% 


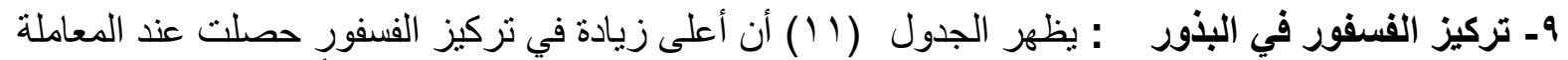

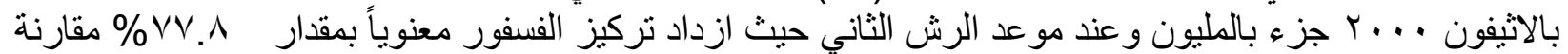

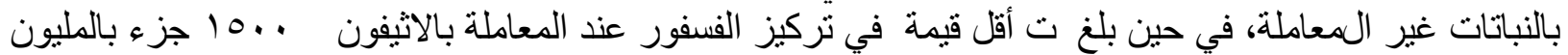

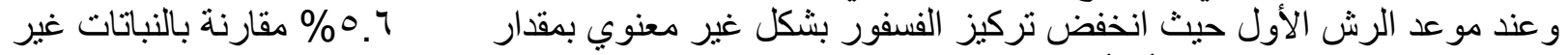

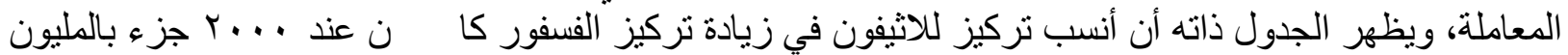

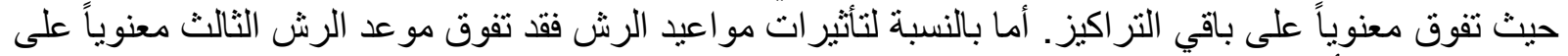

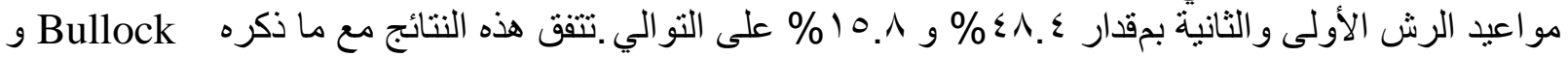

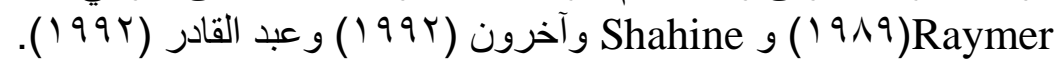

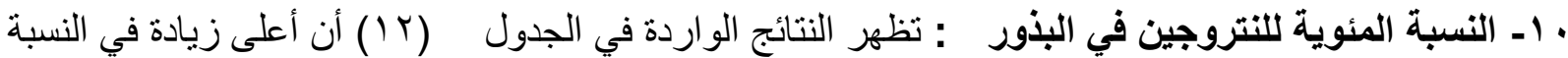

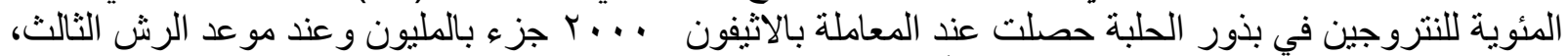

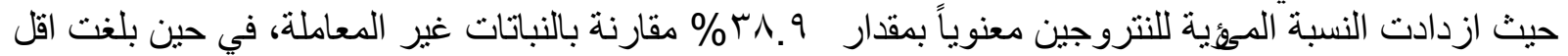

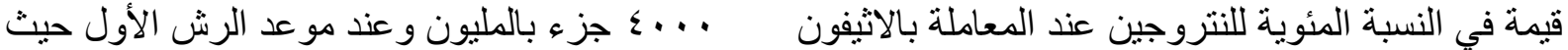

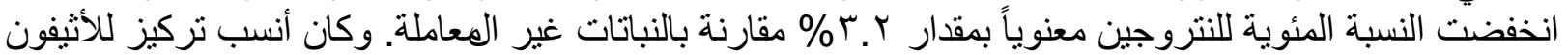

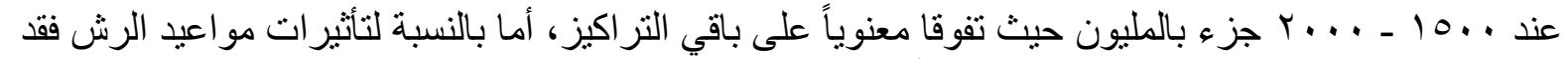

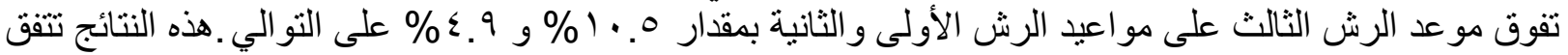

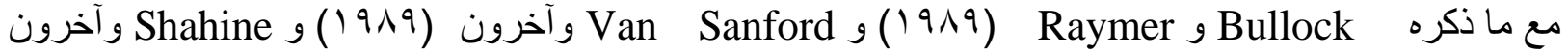

(1994)

الجدول (1 (1) نأثير نر اكيز ومو اعيد رش الاثيفون في صفة تركيز الفسفور ملغم /غم وزن جاف للبذور

\begin{tabular}{|c|c|c|c|c|c|c|c|c|c|c|}
\hline للمواع المعدل & $\varepsilon \ldots$ & ro... & r... & ro.. & r... & $10 \ldots$ & $1 \ldots$ & $0 .$. & صفر & الروش التركيز \\
\hline $\begin{array}{c}8.957 \\
c\end{array}$ & $\begin{array}{c}9.127 \\
\text { gh }\end{array}$ & $\begin{array}{c}9.617 \\
\mathrm{fg}\end{array}$ & $\begin{array}{c}8.271 \\
\mathrm{i}\end{array}$ & $\begin{array}{c}10.67 \\
\mathrm{e}\end{array}$ & $\begin{array}{c}8.202 \\
\mathrm{i}\end{array}$ & $\begin{array}{c}8.134 \\
\mathrm{i}\end{array}$ & $\begin{array}{c}9.206 \\
\mathrm{gh}\end{array}$ & $\begin{array}{c}8.761 \\
\text { hi }\end{array}$ & $\begin{array}{c}\text { A. T/T } \\
\text { hi }\end{array}$ & الاول \\
\hline $\begin{array}{c}11.48 \\
b\end{array}$ & $\begin{array}{c}11.02 \\
\text { de }\end{array}$ & $\begin{array}{c}11.23 \\
\mathrm{de}\end{array}$ & $\begin{array}{c}11.61 \\
\mathrm{~d}\end{array}$ & $\begin{array}{c}9.880 \\
\mathrm{f}\end{array}$ & $\begin{array}{c}15.03 \\
\mathrm{a}\end{array}$ & $\begin{array}{c}12.29 \\
\mathrm{c}\end{array}$ & $\begin{array}{c}11.23 \\
\mathrm{de}\end{array}$ & $\begin{array}{c}12.57 \\
\mathrm{c}\end{array}$ & $\begin{array}{c}8.453 \\
\mathrm{i}\end{array}$ & اني \\
\hline $\begin{array}{c}13.29 \\
\mathrm{a}\end{array}$ & $\begin{array}{c}14.86 \\
\mathrm{a}\end{array}$ & $\begin{array}{c}13.51 \\
b\end{array}$ & $\begin{array}{c}14.74 \\
\mathrm{a}\end{array}$ & $\begin{array}{c}14.86 \\
\mathrm{a}\end{array}$ & $\begin{array}{c}13.34 \\
b\end{array}$ & $\begin{array}{c}14.53 \\
\mathrm{a}\end{array}$ & $\begin{array}{c}12.67 \\
\mathrm{c}\end{array}$ & $\begin{array}{c}12.37 \\
\mathrm{c}\end{array}$ & $\begin{array}{c}8.727 \\
\text { hi }\end{array}$ & الثالث \\
\hline & $\begin{array}{c}11.67 \\
b\end{array}$ & $\begin{array}{c}11.45 \\
\text { bc }\end{array}$ & $\begin{array}{c}11.54 \\
\text { bc }\end{array}$ & $\begin{array}{c}11.80 \\
b\end{array}$ & $\begin{array}{c}12.19 \\
\mathrm{a}\end{array}$ & $\begin{array}{c}11.65 \\
b\end{array}$ & $\begin{array}{c}11.03 \\
\mathrm{~d}\end{array}$ & $\begin{array}{c}11.23 \\
\mathrm{~cd}\end{array}$ & $\begin{array}{c}8.598 \\
\mathrm{e}\end{array}$ & للتركيز \\
\hline
\end{tabular}

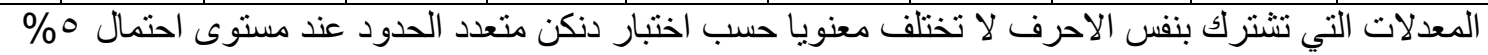

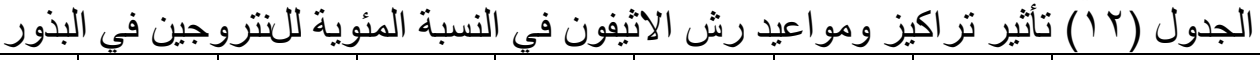

\begin{tabular}{|c|c|c|c|c|c|c|c|c|c|c|}
\hline للمولاع المعدل & $\varepsilon \ldots$ & ro... & r... & Yo.. & r... & $10 \ldots$ & $1 \ldots$ & $0 .$. & صفر & موعد الرش التركيز \\
\hline $\begin{array}{c}3.573 \\
c\end{array}$ & $\begin{array}{c}3.183 \\
1\end{array}$ & $\begin{array}{c}3.250 \\
1\end{array}$ & $\begin{array}{c}3.523 \\
j\end{array}$ & $\begin{array}{c}3.656 \\
g-j\end{array}$ & $\begin{array}{c}3.783 \\
\text { fgh }\end{array}$ & $\begin{array}{c}4.046 \\
\text { de }\end{array}$ & $\begin{array}{c}3.836 \\
\mathrm{fg}\end{array}$ & $\begin{array}{c}3.59 \\
\mathrm{ij}\end{array}$ & $\begin{array}{c}r . r q \\
1\end{array}$ & الاول \\
\hline $\begin{array}{c}3.763 \\
b\end{array}$ & $\begin{array}{c}3.240 \\
1\end{array}$ & $\begin{array}{c}3.300 \\
1\end{array}$ & $\begin{array}{c}3.753 \\
\mathrm{f}-\mathrm{i}\end{array}$ & $\begin{array}{c}3.896 \\
\text { ef }\end{array}$ & $\begin{array}{c}4.383 \\
\text { bc }\end{array}$ & $\begin{array}{c}4.210 \\
\mathrm{~cd}\end{array}$ & $\begin{array}{c}4.116 \\
d\end{array}$ & $\begin{array}{c}3.650 \\
\text { hij }\end{array}$ & $\begin{array}{c}3.333 \\
\mathrm{kl}\end{array}$ & ناني \\
\hline $\begin{array}{c}3.948 \\
\mathrm{a}\end{array}$ & $\begin{array}{c}3.296 \\
1\end{array}$ & $\begin{array}{c}3.496 \\
\mathrm{jk}\end{array}$ & $\begin{array}{c}3.836 \\
\mathrm{fg}\end{array}$ & $\begin{array}{c}4.216 \\
\mathrm{~cd}\end{array}$ & $\begin{array}{c}4.630 \\
a\end{array}$ & $\begin{array}{c}4.490 \\
\mathrm{ab}\end{array}$ & $\begin{array}{c}4.373 \\
\text { bc }\end{array}$ & $\begin{array}{c}3.860 \\
\mathrm{f}\end{array}$ & $\begin{array}{c}3.333 \\
\mathrm{kl}\end{array}$ & الثالث \\
\hline
\end{tabular}




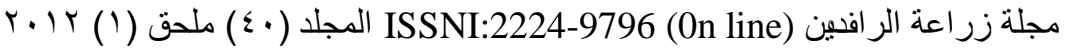

ISSN:1815-316X (Print)

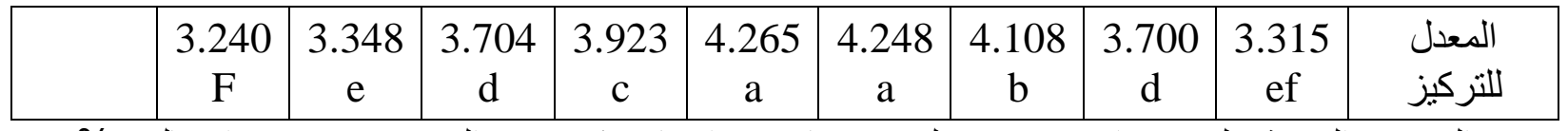

المعدلات التي تشترك بنفس الاحرف لا تختلف معنويا حسب اختبار دنكن متعدد الحدود عند مستوى احتمال 0\%.

\section{Effect of Ethephon on Physiological Growth Parameters And Mineral Component Of Fenugreek (Trigonella foenum-graecum L.)}

Kazwan K.H.Al-Taee

Mosul univ./College of Education/Dep. of Biology

Saad Al-deen M. Al-Hafodhy

Abstract

This study was carried out in green under field condition at department of biology/college of Education for the winter season 2003-2004 to investigate the effect of ethephon concentration $(0,500,1000,1500,2000,2500,3000,3500,4000)$ ppm. a.i. spraying at three growth stages (seedling stage at 30 days, vegetative growth stage at 60 days, beginning flowering growth stage at 90 days) on some growth parameters ( vegetative dray weight, Leaf Area Ratio (L.A.R.), Leaf Area Duration (L.A.D.), Net Assimilation Rate (N.A.R.), BioMass Duration (B.M.D.), Crop Growth Ratio (C.G.R.) and mineral composition ( $\mathrm{N} \%, \mathrm{P} \%, \mathrm{~K} \%$ ) of local fenugreek (Indian helpa). The design which used is R.C.B.D. with three replicates. The highest figures for (vegetative dry weight, leaf area ratio L.A.R., leaf area duration L.A.D., Biomass Duration B.M.D., crop growth ratio C.G.R.) were found at ethephon (1000-1500) ppm. Ethephon at high levels (3500)ppm. increased the value of net Assimilation Rate N.A.R. also the ethephon at (2000) ppm caused highly increased in $\mathrm{N} \%, \mathrm{P} \%$. K\% percent.The first spraying date at seedling period were significantly advanced at all vegetative growth values than other spraying dates.

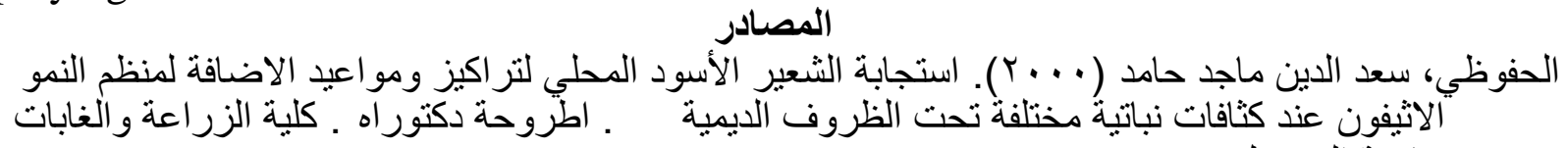

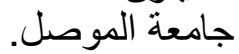

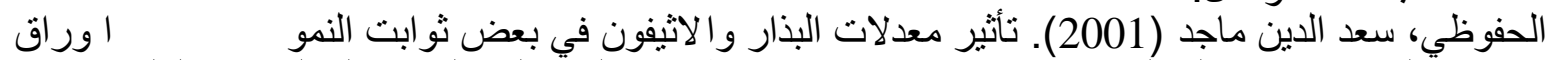

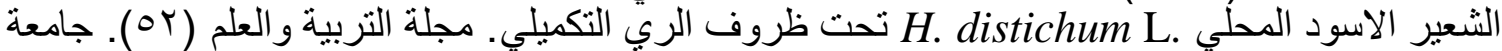

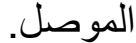

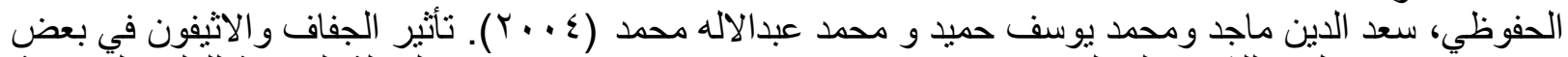

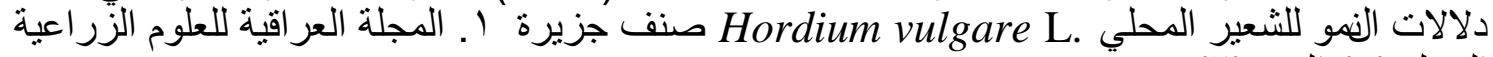

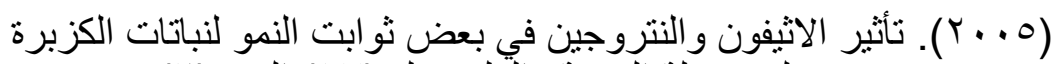

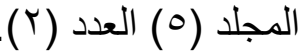

Coriandrum sativum L.

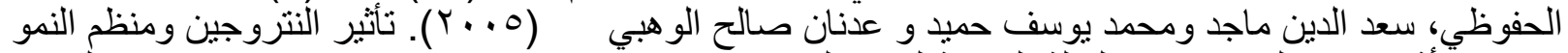

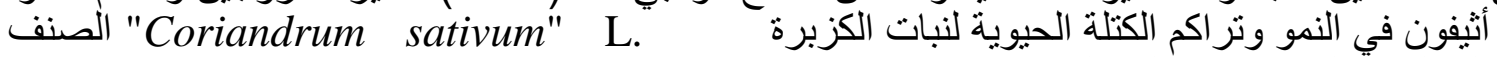

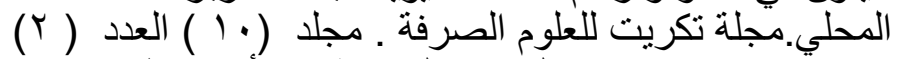

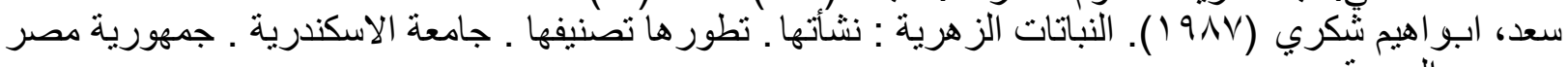
العربية. 


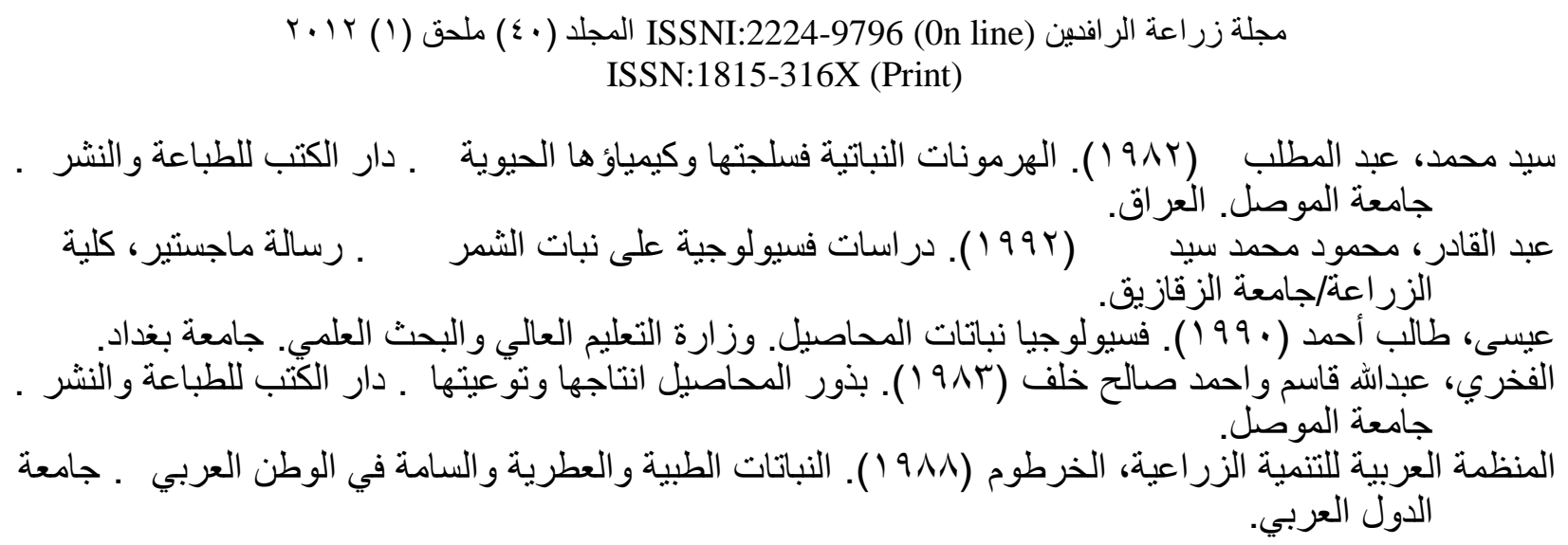

A.O.A.C. (1980) Official Methods of Analysis. Assoc. ossi. Agric. Chemi., Washington, $13^{\text {th }}$ ed.

A.P.H.A. (1985). Standard Methods for the Examination of water \& Wastewater. Amer. Pubi. Heal. Assoc., New York, $16^{\text {th }}$ ed.

Abd-El-Wahab, A. S. (1982). Effect of NPK-supply on growth, yield and on the active principles of some medicinal plants, Ph.D. Thesis. University of Horticulure, Budapest. Hungaria.

Bullock, D. G. and Raymer, P. L. (1989). Growth, grain yield, and tissue Mineral concentration of corn treated with ethephon. Agron. J. 81: 480-483.

Chapman, H. D. and Pratt, P. F. (1961). Methods of Analysis for Soils. Plants and Water Univ. of Calif., Div. of Agric, Sci., 39: 309.

Duncan, D. B. (1955). Multiple Range and Multiple F test. Biometrics 11 : 1-42.

Foster, K. R.; Reid, D. M.; and Taylor, J. S. (1991). Tillering and yield responses to ethephon in three barley cultivars. Crop Sci. 31(1) 130-134.

Gamrod, E. E (2003). Flowerring control and production of Strobilanthes dyerianus Mast of science in Horticalture. Black burg. Virginia.

Harper, J. L. (1977). Population biology of plants. Academic press. London, P: 305-345.

Hoogenboom, G.; Huck, M. G. and Peterson ,C. M. (1986). Measured and simulated drought stress effect on daily shoot and root growth rates of Soybean. Nether. J. Agric. Sci. 34: 497-500.

Hugi, K. and Keller, E. R. (1990). Can the yielding ability of faba beans be improved? Landwirts chaft-Schweiz. 3(6): 273-178.

Hunt, R. (1978). Plant Growth Analysis. London. Edward Arnold.

Kasele, I. N.; Nyirenda, F.; Shanahan, G. F.; Nielsen, C. D. and D'Andria, R. (1994). Ethephon alters Corn growth, water use, and grain yield under drought stress. Agron. J. 86(2): 283-288.

Lauer, J. G. (1991). Barley response to plant density and ethephon. Agron. J. 83(6): 968973.

Norberg, O. S.; Mason ,S. C. and Lowry, S. R. (1988). Ethephon influence on harvestable yield, grain Quality, and lodging of Corn. Agron. J. 80: 768-772.

Norberg, O. S.; Mason, S. C. and Lowry, S. R. (1989). Ethephon alteration of cron plant morphology. Agron. J. 81: 603-609.

Radford, P. J. (1967).Growth analysis formula ,their use and abuse. Crop Sci. 7:71-76.

Rosenberg, N. J.; Blad, B. L. and Verma ,S. B. (1983). Microclimate: the biological environment 2nd Ed. Wiley - inter science, New York.

Shahine, A. H.; El-Desouky, S. A.; A.; Abd-El-Doyern, H. M. and Wanas, A. (1992). Response of fenugreek (Trigonella foenum -graceum L.) and pea (Pisum sativum L.) to foliar spray with some growth regulators. 11-flowering, dry weight, seed 


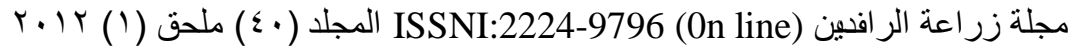 ISSN:1815-316X (Print)}

production and its organic matter content. Annals-of-agriculture 1-scince,Moshtohor. 30(2): 755-775.

Shil-nikova, V. K.; Volobuera, O. G. (1992). Effectiveness of inoculating pea seeds when the plants are treated with growth regulator. Izvestiya-TimiryaZeveskoiSel'skohozyaistvennoi-Akademii. No.1: 85-91.

Simmons, S. R.; Oelke, E. A.; Wiersma, J. V.; Lenschen, W. E. and Warnes, D. D. (1988). Spring wheat and barley responses to ethephon. Agrion. J. 80: 829-834.

Soybean. J. prod. Agric. American Society of Agronomy 1990. 3(4): 564-568. (C. F. Agricola Abstract).

Teyker, R. H.; Litwiller, G. D. and Ahmed, H. I. (1990). Effect of ethephon on morphology, yield and lodging in Williams 82.

Van Sanford, D. A.; Grove, J. H; Grabau ,L. J. and Mackown ,C. T. (1989). Ethephon and nitrogen use in winter wheat. Agron. J. 81(6): 951-954. 